\title{
Wild plants used for food by Hungarian ethnic groups living in the Carpathian Basin
}

\author{
Andrea Dénes ${ }^{1 *}$, Nóra Papp ${ }^{2}$, Dániel Babai ${ }^{3}$, Bálint Czúcz ${ }^{4}$, Zsolt Molnár ${ }^{4}$ \\ ${ }^{1}$ Natural History Department, Janus Pannonius Museum, Box 158, 7601 Pécs, Hungary \\ 2 Department of Pharmacognosy, University of Pécs, Rókus 2, 7624 Pécs, Hungary \\ ${ }^{3}$ Centre for Humanities, Hungarian Academy of Sciences, Országház 30, 1014 Budapest, Hungary \\ ${ }^{4}$ Centre for Ecology, Hungarian Academy of Sciences, Alkotmány 2-4, 2163 Vácrátót, Hungary
}

\section{Abstract}

A list of plant species used for food in Hungary and among Hungarian ethnic groups of the Carpathian Basin during the 19th and 20th centuries was compiled from 71 ethnographic and ethnobotanical sources and a survey among contemporary Hungarian botanists. Species used as food, spice, beverage or occasional snacks were collected. Sources mention 236 plant species belonging to 68 families. Most wild fleshy fruits (mostly Rosa, Rubus, Cornus, Ribes, Vaccinium spp.), dry fruits and seeds (Fagus, Quercus, Corylus, Castanea, Trapa spp.), several green vegetables (e.g. Rumex, Urtica, Humulus, Chenopodiaceae spp., Ranunculus ficaria), bulbs and tubers (Lathyrus tuberosus, Helianthus tuberosus, Chaerophyllum bulbosum, Allium spp.) used for food in Europe, are also known to be consumed in Hungary. A characteristic feature of Hungarian plant use was the mass consumption of the underground parts of several marsh (e.g. Typha, Phragmites, Sagittaria, Alisma, Butomus, Bolboschoenus spp., as well as the endemic Armoracia macrocarpa) and steppe species (e.g. Crambe tataria, Rumex pseudonatronatus). Consuming wild food plants is still important among Hungarians living in Transylvania: even nowadays more than 40 species are gathered and used at some locations.

Keywords: ethnobiology, historical ethnobotany, wild green vegetables, wild edible plants, tree saps

\section{Introduction}

In the last two decades several comprehensive surveys and reviews were performed in many European countries on wild plant use. Wild plants formed important parts of our ancestors' diet in a multitude of ways (e.g. as bread ingredients, vegetables, fruits, spices, snacks or beverages); essential in severe times, supplementary otherwise [1-3]. Revitalization of traditional practices is timely for ecology, economy and nutrition biology. Nevertheless, even though Hungary is no poorer in traditions of wild plant use than other parts of Europe, no broad-scale review has been undertaken for Hungary in this respect, yet.

Information on the human consumption of wild plant species is widely scattered among the ethnographic and botanic literature, mostly in Hungarian language. Publications on gathering economy, traditional nutrition, shepherding, forest goods utilization, and on the hunting-fishing-gathering "pákász"

*Corresponding author. Email: denes.andrea@jpm.hu

This is an Open Access digital version of the article distributed under the terms of the Creative Commons Attribution 3.0 License (creativecommons.org/licenses/by/3.0/), which permits redistribution, commercial and non-commercial, provided that the article is properly cited.

(c) The Author(s) 2012 Published by Polish Botanical Society lifestyle in the former wetlands of the floodplains of the large Hungarian rivers are known from the end of 18th century on (e.g. [4]), and they became frequent from the second half of the 20th century. Publications of traditional research on medicinal uses of plants [5-8] and ethnobotany [9-11] also mention wild food plants, but a review concentrating on wild food plants is still missing.

In this work we systematically review and compile information on the utilization of wild food plants in the Hungarian speaking regions of the Carpathian Basin from the Hungarian ethnographic and ethnobotanical publications. Our study aims at compiling a knowledge base on wild food plants consumed in Hungary and by the Hungarians living in other countries of the Carpathian basin, containing information on the species, as well as the modes of use. As the Carpathian Basin is dominated by alluvial floodplains located on the margin of the vast Eurasian steppe regions, Hungarian traditional plant use might also include previously undocumented usage of wetland and steppe plant species, which can potentially add some new aspects to the existing knowledge on traditional plant use in Europe.

\section{Material and methods}

\section{Flora, vegetation and history of the Carpathian Basin}

The Carpathian Basin is the contiguous floodplain area of the Danube and Tisza Rivers encircled by the Carpathian 
Mountains in Central Europe. Containing an extensive transitional zone between European deciduous forests and the Eurasian steppe biome the study area harbors a particularly diverse vegetation, which is also acknowledged by the European Union by classifying the majority of this region into a singular biogeographic zone, the Pannonian biogeographic zone.

The periphery of the Carpathian Basin including the Carpathians can be characterized mostly by and alpine and subalpine vegetation, coniferous forests, which turn into broadleaved deciduous forest at lower elevations. The central part of the basin is dominated by continental forest-steppes, although only remnants of salty and sand steppes had survived to date. Gallery forests and wetlands on the floodplains of the two large rivers, Danube and Tisza, and their tributaries played a determining role in vegetation development and also in people's lives till their regulations in the second part of the 19th century. Presently their former area, as well as the majority of former steppe vegetation, is dominated by agriculture [12]. The flora of Hungary consists of 2600 species including many steppe species, whereas the flora of Transylvania is also about 2600 with significant proportions of boreal and alpine species [13]. The Carpathian Basin altogether harbors 3360 species. In addition to the dominant Eurasian, continental and European species, southern, submediterranean and Balcanic elements amount to $20 \%$.

As a consequence of Hungarian history, there are Hungarian ethnic groups living in all countries of the Carpathian Basin. Due to ecological, historical and economic reasons, ethnicities separated from the mother country often preserve their traditions better, even archaic ones, so researchers prefer to conduct ethnographic and ethnobotanic studies among Hungarians living in Romania, Slovakia, Ukraine, Serbia and Croatia.

\section{Research history}

Historical records on the use of wild plants in Hungary are known since the Medieval period (16th century). Herbal knowledge of wise women and monks, and works of former botanists as Clusius (1526-1609), Beythe (1532-1612), Kitaibel (1758-1817), Borbás (1844-1934), and Dégen (1866-1934) are usually reviewed by ethnographers studying the gathering economy of the Carpathian Basin. Detailed records on edible plants from the end of the 18th century, can be found in the manuscript diaries of Kitaibel, reviewed recently by Molnár [4]. Rapaics [14] had published an overview on the history of food plants, going back to pre-Medieval times.

A complete list of former publications can be found in the reviews of Gunda $[15,16]$, a key person of ethnographical research of gathering economy in Hungary. Reviews of folk nutrition [17-19] generally include more or less detailed overviews of wild plants collected for food [9,17-19]. Plants consumed during famines were studied by Rapaics [14], Györffy [20], Gunda [15,16], and Molnár [21]. The published studies collectively cover almost the entire Carpathian Basin, the most thoroughly explored regions being Transylvania [sensu lato, including e.g. Gyimes (Ghimeș) and Máramaros (Maramureș)] and the southern foothills of the Northern Carpathians [Nógrád, Heves, Borsod, and Zemplén counties as well as the Gömör (Gemer, Slovakia) region]. Nevertheless, not all papers provide a comprehensive ethnobotanical "wild food plant" survey of the studied regions - there are several papers, where the focus is on other aspects of traditional living, with only sporadic mention of a few wild plants.

\section{Methods}

We compiled a list of plant species used for food based on 71 ethnographic and ethnobotanical publications in Hungarian language. Most of the studied papers describe traditional ecological knowledge of Hugarian speaking ethnic groups living in the Carpathian Basin, even though there might be exceptions, e.g. some papers included wild food plants used by other ethnic groups (e.g. Slovaks) living in Hungary, while other papers include traditional plant use of Hungarians living even beyond the Carpathians (e.g. Hungarian refugees from Bukovina, whose plant use included ancient elements from their old home country as well as newer ones from their new home, Hungary). The studied publications explore the following regions: Dunántúli dombvidékek (SW Hungary: Örség, Somogy, Zala, Tolna, Baranya) [22-29]; Kisalföld (NW Hungary and SW Slovakia) [30,31]; Déli-Alföld (SW Hungary and N Croatia) [32-35]; Dunántúli középhegység (mountains in W Hungary) [36,37]; Északi középhegység (Carpathian foothills in N Hungary) [38-47]; Gömör (Gemer, S Slovakia) [48-52]; Kárpátalja (Zakarpatska Oblast, W Ukraine) [53,54]; the Great Hungarian Plain [55-62]; Vajdaság (Voivodina, N Serbia) [63]; Erdély (Transylvania, Romania) [64-75]; and Bukovina (N Romania - collected from refugees settled down in Hungary) [76,77] (Fig. 1). This literature review was complemented by a survey among 34 Hungarian botanist on the wild food plants which they had collected and consumed in their childhood [78].

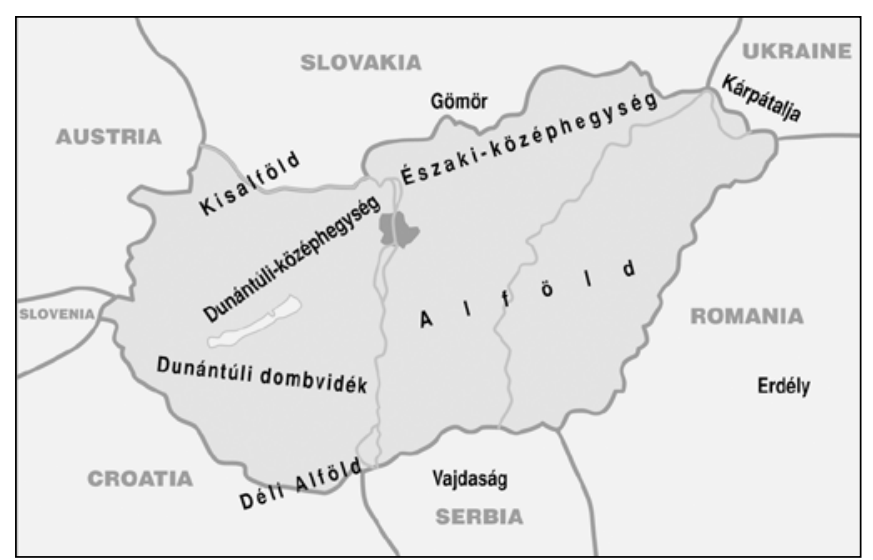

Fig. 1 Map of the study area including the geographic names used in the text.

The oldest source is from the end of the 18th century [4]; the most recent ones are up-to-date ethnobotanical surveys collected in Ghimeș, Transylvania and Hortobágy, Hungary $[11,74,75]$. In addition to local traditional plant names, most publications also mention the official Hungarian and/or the scientific (genus or species) Latin names of the food plants, even though these identifications can be easily mistaken if the local traditional names are similar to the official Hungarian name of another taxon. Such misidentifications are unfortunately typical in a part of the ethnography literature, nevertheless a good botanical knowledge, the descriptions of the plants discussed (if supplied), and the comparison of the different sources can effectively help to correct misidentifications [79]. In our work we corrected all obvious misidentifications which could be easily corrected, and omitted all records which were clearly invalid, but no unambiguous correction was available. Nevertheless, most of the species are mentioned in several 
publications, which reduces the uncertainties, particularly in the case of plant uses which were also documented by botanists.

In the reviewed papers we focused on plants which were collected from the wild and consumed as food or food ingredient (including spices, beverages, occasional snacks, etc.). This definition excludes medical plants (consumed only for their health impacts), and plants collected only for non-food use (e.g. dye, timber, etc.). On the other hand, we included gathering from spontaneous populations of escaped and naturalized cultivated plants and non-native invasives.

\section{Results}

We found altogether 235 plant species belonging to 67 families which were mentioned from the study area. We found 216 species in the literature survey, whereas contemporary Hungarian botanists (34 data providers) mentioned 91 taxa (the overlap between the two sets was 71 species). The species are listed in detail in Tab. 1 with their local names as mentioned in the sources, and their documented modes of usage. In order to provide an indicator for record uncertainty, species data published only by ethnographers are marked with “»” in Tab. 1.

We found that it is the species of the Rosaceae family (36 species) which are consumed most often; other frequently used families include Asteraceae, Lamiaceae, Liliaceae (sensu lato, before the APG division of the family), Fabaceae and Apiaceae. The list contains 36 trees, 27 shrubs, 4 dwarf shrubs and 169 herbaceous species. Green aboveground parts (leaves, young shoots, buds, and sometimes the whole plant) of 98 species were consumed (mainly Apiaceae, Lamiaceae, Liliaceae). Flowers of 39 species (mainly Asteraceae, Boraginaceae, and Lamiaceae) and fruits/seeds of 74 species (mainly Rosaceae, Grossulariaceae, and Ericaceae) were eaten. Underground parts - roots, rhizomes, tubers, bulbs - of 23 species (mainly Liliaceae, Apiaceae, Asteraceae, and Brassicaceae) were used. Saps of 8 species and dried saps (resins, gums) of 4 species were consumed or chewed. In several cases two or three parts of the same species were consumed, e.g. Rosa gallica: flowers, fruits, leaves; Fagus sylvatica: fruits, leaves and sap; Sambucus nigra: flower, fruit; Taraxacum spp.: leaves, flowers; Fragaria spp.: fruits and leaves.

\section{Green vegetables}

Green parts - mainly young spring shoots or young leaves - of 51 species were prepared raw for salad, or cooked for use in soup or sauce. Species used in most regions include Rumex acetosa, Urtica dioica, Humulus lupulus, Ranunculus ficaria, and Allium spp. Larger leaves are often used in regional dishes as a wrapping for some meaty stuffing, e.g. Armoracia rusticana, Tussilago farfara and Fallopia spp. Young shoots of Typha spp. and Phragmites australis used to be consumed as a salad in the Sárköz region of Hungary, a tradition abandoned long time ago. Sixteen species including Anthriscus cerefolium, Glechoma hederacea, Thymus spp., and Verbena officinalis were used as spice, pickling or preservative. Satureja alpina as a spice is mentioned from the early 18 th century. The green parts of several plants were used to prepare a refreshing tea or as syrup (e.g. a "pine honey" made from the buds of Pinaceae species); more interesting uses include making candy from Melittis melissophyllum [76], or Fagus leaves. Babies unable to suck were fed with pressed nettle (Urtica) sap (I. Németh personal communication). Some species (e.g. Oxalis acetosella, Galium verum) were used in the past as curdling agents. Herdsmen chewed Plantago lanceolata leaves in order to clean their teeth (I. Németh personal communication.). Eating Lemnaceae species in famine is mentioned only from the Drava floodplain.

\section{Flowers}

Even though sucking nectar and eating flowers or inflorescences is a widespread and delightful occupation of children, major food or beverage products are rarely made from flowers. As an exception, flowers of Sambucus nigra or Robinia pseudacacia are commonly used for making refreshing drinks or fried into pancakes they can serve as popular dishes. Unique is the use of inflorescences of Carlina acaulis as a green vegetable. Flowers of Humulus lupulus and Robinia pseudacacia were added to sourdough in many places. In Gömör, there used to be a tradition of making "flower wines" from Robinia or Taraxacum flowers ("pimpóbor”) [50].

\section{Fruits and seeds}

Eating and processing wild fruits of many species is a widespread, still a living tradition in Hungary. Fragaria, Sambucus, Rubus, Crataegus, Vaccinium, Ribes, Rosa spp. and Cornus mas have been eaten fresh, baked into cakes, prepared as beverages, or dried for a later use for long time. With the advent of cheap sugar additional preservation techniques became available including syrup and jam production [17]. Some fruits (Malus, Pyrus, Vaccinium vitis-idaea, Streptopus amplexifolius) were collected unripe; they were ripened in the attic, in hay, or in the sun. Formerly cider and vinegar were fermented from the fruits of Malus sylvestris. A traditional fruit brandy called "pálinka" is traditionally distilled after fermentation from many fruits even today. To improve taste and color fruits and spices can also be added to "pálinka" after distillation ("ágyaspálinka"). Fruit cider and brandy production was particularly important in regions without extensive grape productions. In Gömör cider was fermented from almost all wild fruits. Rarely consumed fruits include Streptopus amplexifolius, Viburnum spp. and Cornus sanguinea, which are considered slightly toxic by some sources but were still documented as consumed by trustworthy publications. Several dry fruits (nuts and seeds) were also widely collected. In addition to the nuts being still economically significant (Corylus avellana, Juglans regia, and Castanea sativa), Fagus sylvatica seeds were also eaten raw or roasted. Ground Fagus acorns, as well as Quercus acorns after leaching, were also used as coffee substitutes and flour in famine times. In addition to acorns, famine flour ingredients also included dried and ground Crataegus, Rosa and Trapa fruits, Glyceria seeds and Corylus buds. According to Kitaibel's data from the end of the 18th century, oil was pressed from Sisymbrium altissimum, Brassica nigra, and Fagus sylvatica seeds. Staphylea pinnata nuts used to be a kids' snack; cooked unripe fruits of Daphne mezereum were used as black pepper substitute in the time of Kitaibel, at the beginning of the 1800s. Carum carvi used to be a widespread spice not only for dishes, but also for palinka and tea. Food and wine were coloured, e.g. with Phytolacca americana; while shiny seeds, e.g. Vicia spp. and Lathyrus aphaca were used for decorating cakes.

\section{Underground parts}

Underground parts of of several wild plants were among the most important staple foods during famines. Bulbs, tubers or rhizomes of Alisma plantago-aquatica, Bolboschoenus 
Tab. 1 List of wild food plants used by Hungarians in the Carpathian Basin.

\begin{tabular}{|c|c|c|c|c|}
\hline Scientific name & Local names mentioned & Parts used & Mode of use & Reference No. \\
\hline Abies alba Mill. & fehérfenyő & VEG, FLO & $\begin{array}{l}\text { brandy (palinka) was distilled from buds } \\
\text { and young cones }\end{array}$ & {$[74]$} \\
\hline Acer campestre L. & kokasfa, kokastorufa, juhar & FRU & immature fruits were sucked as a snack & {$[23,78]$} \\
\hline Acer pseudoplatanus L. & jávor, jávorfa, hegyi juhar & SAP & beverage & [19] \\
\hline Acer sp. & juhar, jávor, jávorfa & SAP & beverage & {$[9,52,69,72]$} \\
\hline Acorus calamus L.* $\dagger$ & kálmos & SUB & spice for liqueur & [29] \\
\hline Aesculus hippocastanum L. $\dagger$ & vadgesztenye & FRU & seeds as coffee substitute & {$[19,78]$} \\
\hline Agrimonia eupatoria L. & $\begin{array}{l}\text { párlófü, tüdőfü, bojtorján, bojtorván, bojtorvány, } \\
\text { repcsik }\end{array}$ & VEG & tea & {$[19,43,67]$} \\
\hline Alchemilla spp. & palástfü, harmatfü & VEG & raw as salad & {$[74]$} \\
\hline Alisma plantago-aquatica $\mathrm{L}$. & type of bengyele, bakacs & SUB & food in famine and for herdsmen & {$[16,21]$} \\
\hline $\begin{array}{l}\text { Allium atroviolaceum } \\
\text { Boiss.* }^{*}\end{array}$ & mezei fokhagyma & VEG, SUB? & raw & {$[57]$} \\
\hline Allium obliquum $\mathrm{L}$. & turkesztáni hagyma & VEG, SUB? & raw & {$[65]$} \\
\hline Allium oleraceum $\mathrm{L}$. & érdes hagyma & VEG, SUB? & raw and as spice & {$[21]$} \\
\hline Allium rotundum $\mathrm{L}$. & n.d. & VEG, SUB? & eaten & {$[65]$} \\
\hline Allium scorodoprasum L. & $\begin{array}{l}\text { hagyma, vadhagyma, kígyóhagyma, vad } \\
\text { fokhagyma }\end{array}$ & VEG & $\begin{array}{l}\text { young leaves were eaten; also used as spice } \\
\text { like garlic }\end{array}$ & {$[4,19,41,57,78]$} \\
\hline Allium ursinum $\mathrm{L}$. & $\begin{array}{l}\text { vadfokhagyma, medvehagyma, } \\
\text { medvefokhagyma, vad hagyma, medvesósdi, } \\
\text { sorhajma, salama }\end{array}$ & VEG & salad, vegetable, spice (put into sausage) & $\begin{array}{l}{[27,41,65,70} \\
73-75]\end{array}$ \\
\hline Allium victorialis $\mathrm{L}$. & győzedelmes hagyma & VEG, SUB? & eaten & {$[4]$} \\
\hline Allium vineale $\mathrm{L}$. & n.d. & VEG, SUB & leaves and bulbs were eaten & {$[78]$} \\
\hline Allium sp. & hagyma & FLO & $\begin{array}{l}\text { small onions in inflorescence were } \\
\text { children's snack }\end{array}$ & {$[78]$} \\
\hline Alopecurus pratensis L. & gombos ecsetpázsit & VEG & stem was children's snack & {$[78]$} \\
\hline Anchusa officinalis L. & n.d. & VEG & eaten as salad, scalded with vinegar & {$[65]$} \\
\hline Anthriscus cerefolium (L.) & zamatos turbolya, turbolya & VEG & green spice for soups and vegetables; & {$[65,78,86]$} \\
\hline Hoffm. subsp. trichosperma & & & rarely mentioned in folk literature, but & \\
\hline (Spr.) Arc. (Chaerophyllum & & & in a cookbook from the end of the 19th & \\
\hline trichosporum) \& A. sylvestris & & & century A. cerefolium was mentioned as & \\
\hline (L.) Hoffm. (Ch. sylvestre) & & & soup and spice & \\
\hline Amaranthus spp. & paraj & VEG & whole plant was consumed & {$[78]$} \\
\hline Arctium lappa L. & $\begin{array}{l}\text { bojtorján, keserütorzsa, burusztujlapi, keserü- } \\
\text { torzsa lapi, büdös lapi, parti lapi, büdös levél, } \\
\text { parti fü, lapu, bogáncs }\end{array}$ & SUB & peeled and eaten & {$[77,78]$} \\
\hline $\begin{array}{l}\text { Armoracia macrocarpa (W. } \\
\text { et K.) Baumg. }\end{array}$ & torma (debreceni torma) & VEG, SUB & $\begin{array}{l}\text { VEG: soup, vegetable; SUB: spice for } \\
\text { pickles }\end{array}$ & {$[14,59,62]$} \\
\hline $\begin{array}{l}\text { Armoracia rusticana G. } \\
\text { Gaertnn. B. Mey. et Schreb } \\
\text { (A. lapathifolia Usteri) }\end{array}$ & torma, tormalapu & VEG, SUB & $\begin{array}{l}\text { SUB: side dish for meat, VEG: leaves: } \\
\text { soup, sauce; meaty stuffing filled into them }\end{array}$ & $\begin{array}{l}{[9,23,36,42,52} \\
53,62,76]\end{array}$ \\
\hline $\begin{array}{l}\text { Arrhenatherum elatius (L.) J. } \\
\text { et C. Presl. }\end{array}$ & fü & VEG & stem was children's snack & {$[78]$} \\
\hline Artemisia absinthium L. $\dagger$ & fehér üröm, fejér üröm & VEG & spice for brandy (palinka) and wine & {$[30,62,77]$} \\
\hline Asperula odorata L.* & szagos müge & VEG & spice for liqueur & {$[30]$} \\
\hline Atriplex patula L. & vad laboda, sós paréj, fodros paréj & VEG & soup & {$[77]$} \\
\hline Atriplex tatarica L. & fehér laboda & VEG & $\begin{array}{l}\text { raw: salad, cooked: soup, former } \\
\text { herdsmen prisoners of war put it into hot } \\
\text { soup in Siberia }\end{array}$ & {$[11,71]$} \\
\hline Berberis vulgaris $\mathrm{L}$. & fajisóska, nyúlsom, sóska, sóskaborbolya & VEG, FRU & $\begin{array}{l}\text { VEG, FRU: snack; FRU: substitute for } \\
\text { vinegar }\end{array}$ & {$[36,67,78]$} \\
\hline $\begin{array}{l}\text { Betula pendula Roth (B. } \\
\text { verrucosa Roth) }\end{array}$ & nyír, nyírfa, májfa & SAP, VEG & $\begin{array}{l}\text { SAP: fresh beverage; wine and vinegar } \\
\text { were fermented from it; herdsmen of } \\
\text { Transylvania and Zemplén used it for } \\
\text { inoculation of milk. VEG: substitute for } \\
\text { tea }\end{array}$ & $\begin{array}{l}\text { SAP: [9,22-24, } \\
\text { 29,30,38,41-44, } \\
48,50,52,53, \\
\text { 56,58,63,65,66, } \\
\text { 69,72,74]; VEG: } \\
\text { [7] }\end{array}$ \\
\hline Bolboschoenus maritimus & csatak, zsiók, zsiku, zsióka & SUB & food in famine and for herdsmen & {$[14,16]$} \\
\hline
\end{tabular}




\begin{tabular}{|c|c|c|c|c|}
\hline Scientific name & Local names mentioned & Parts used & Mode of use & Reference No. \\
\hline $\begin{array}{l}\text { Brassica nigra }(\mathrm{L} .) \text { Koch } \\
\text { (Sinapis nigra } \mathrm{L} .)\end{array}$ & franciamustár & FRU & oil was pressed from it & [4] \\
\hline Bromus sterilis $\mathrm{L}$ & vadzab & VEG & stem was children's snack & [78] \\
\hline Bunias orientalis $\mathrm{L}$. & borsoslenke, borsos lenkő, rákányéc, szümcső & VEG & soup and salad in spring & {$[9,74,75,78]$} \\
\hline Butomus umbellatus $\mathrm{L}$. & alacs, elecs, elecske & SUB & food in famine times and for herdsmen & {$[14,16,21]$} \\
\hline Campanula persicifolia $\mathrm{L}$. & kupa, tőcsérvirág, vadcsengő & FLO & snack for children & [9] \\
\hline $\begin{array}{l}\text { Capsella bursa-pastoris (L.) } \\
\text { Medik. }\end{array}$ & pásztortáska & VEG, FLO & $\begin{array}{l}\text { young flowering shoots were snacks for } \\
\text { children }\end{array}$ & {$[74,78]$} \\
\hline Cardamine amara $\mathrm{L}$. & vízitorma & VEG & snack in early spring & [70] \\
\hline Cardamine pratensis $\mathrm{L}$. & n.d. & VEG & n.d. & [73] \\
\hline Carlina acaulis $\mathrm{L}$. & bábakalács, bábakonty, kontybába, kenyérvirág & FLO & $\begin{array}{l}\text { inner part of inflorescence was a raw } \\
\text { snack }\end{array}$ & {$[9,19,74,75]$} \\
\hline Carpinus betulus $\mathrm{L}$. & gyertyán & VEG, SAP & $\begin{array}{l}\text { SAP: fresh beverage. VEG: leaves as a } \\
\text { spring snack }\end{array}$ & $\begin{array}{l}\text { SAP: }[9,23,39] \\
\text { VEG: }[22]\end{array}$ \\
\hline $\begin{array}{l}\text { Carex elata ALL. \& Carex } \\
\text { spp. }\end{array}$ & sás, limbus & VEG & leaves and stems were children's snack & [78] \\
\hline Carum carvi $\mathrm{L}$. & $\begin{array}{l}\text { keménmag, kemény, kömény, kömén, kümén, } \\
\text { köminy, kömin, kömind, köménd, }\end{array}$ & FRU & $\begin{array}{l}\text { spice for rye bread, soups and roasted } \\
\text { meat; brandy (palinka) with honey } \\
\text { and cumin was a traditional drink in } \\
\text { Transylvania }\end{array}$ & $\begin{array}{l}{[7,9,19,36,37} \\
41,52,67,70,71 \\
74-78]\end{array}$ \\
\hline Castanea sativa Mill. & szelidgesztenye, geszkenye, geszkönye & FRU & roasted or cooked for sweets and cakes & $\begin{array}{l}{[23,28-30,36,} \\
37,78]\end{array}$ \\
\hline Celtis occidentalis $\mathrm{L}$. & $\begin{array}{l}\text { ostorfa, zsidómeggy, zsidócseresznye, } \\
\text { madárbogyó, gelegenye }\end{array}$ & FRU & children's snack & [78] \\
\hline Centaurium erythraea Rafn. & cintória, ezerfü & VEG & $\begin{array}{l}\text { raw as an appetizer; spice for brandy } \\
\text { (palinka); tea }\end{array}$ & {$[26,30,57,74]$} \\
\hline $\begin{array}{l}\text { Cerasus avium } \text { (L.) Mönch } \\
\text { (Prunus avium) }\end{array}$ & cseresznye, vadcseresznye, vadcserösznye & $\begin{array}{l}\text { VEG, FRU, } \\
\text { SAPs }\end{array}$ & $\begin{array}{l}\text { VEG: leaf was spice for pickles. FRU: } \\
\text { eaten raw and dried; brandy (palinka) was } \\
\text { distilled from it; SAPs: gum chewing }\end{array}$ & $\begin{array}{l}{[19,22,23,26,} \\
27,29,37,38,40 \\
47,52,57,68,70 \\
71,74,75,78]\end{array}$ \\
\hline $\begin{array}{l}\text { Cerasus vulgaris Mill. subsp. } \\
\text { acida (Dumort.) Dostal } \\
\text { (Prunus cerasus L.) }\end{array}$ & vadmeggy & FRU & raw snack & {$[52,78]$} \\
\hline Chaerophyllum bulbosum $\mathrm{L}$. & $\begin{array}{l}\text { baraboj, bubályka, bobályka, bubolyicska, } \\
\text { buboicska, bóbiska, mogyorófü, turbolya, } \\
\text { csemegebürök, trombujka, mogyorós baraboly, } \\
\text { baraboi, fődibarabój, mihályka, mihálka, } \\
\text { Mihályka monya }\end{array}$ & SUB & $\begin{array}{l}\text { eaten raw by children, also by adults, like } \\
\text { carrot }\end{array}$ & $\begin{array}{l}{[9,14,42,53,58} \\
59,62,65,67 \\
70,75]\end{array}$ \\
\hline Chenopodium album $\mathrm{L}$. & laboda, cigara & VEG & salads and vegetable & {$[19,65,78]$} \\
\hline Cichorium intybus $\mathrm{L}$. & katáng, vad cikória, katángkóró & SUB & $\begin{array}{l}\text { grated and roasted as a coffee substitute; } \\
\text { used even today in Transylvania }\end{array}$ & {$[14,74,78]$} \\
\hline Cirsium arvense (L.) Scop. & tüvis, tövis & VEG & $\begin{array}{l}\text { young spring shoots gathered for soup; } \\
\text { peeled stems eaten raw }\end{array}$ & {$[9,78]$} \\
\hline Cirsium canum (L.) All. & bojhos tövis, ökörlevél, ökörnyelv & VEG & young leaves cooked for soup & {$[9,73]$} \\
\hline $\begin{array}{l}\text { Cirsium oleraceum (L.) } \\
\text { Scop. }\end{array}$ & káposztás acat & VEG & young leaves were eaten & [21] \\
\hline Convolvulus arvensis $\mathrm{L}$. & szulák, győtény & FRU, VEG & $\begin{array}{l}\text { FRU: seeds were famine food; VEG: } \\
\text { children's snack }\end{array}$ & {$[16,21,78]$} \\
\hline Cornus mas $\mathrm{L}$. & som, sum & FRU & $\begin{array}{l}\text { sour jam eaten with meat; syrup; dried; } \\
\text { brandy (palinka) distilled from it }\end{array}$ & $\begin{array}{l}{[19,26,29,30} \\
36-42,46-52 \\
57,62,65,70 \\
72,78]\end{array}$ \\
\hline Cornus sanguinea L. $\dagger$ & somfa & FRU & $\begin{array}{l}\text { jam, syrup and brandy was made from it } \\
\text { (even though some consider it poisonous) }\end{array}$ & [19] \\
\hline Corylus avellana $\mathrm{L}$. & $\begin{array}{l}\text { magyarófa, mogyorófa, mogyoró, fáin magyaró, } \\
\text { fájimogyoró, fájimagyaró, monyaru, monyaró, } \\
\text { magyaru }\end{array}$ & FRU, VEG & $\begin{array}{l}\text { FRU: baked into cakes, snack; VEG: buds: } \\
\text { ground for flour substitute in famine }\end{array}$ & $\begin{array}{l}{[19,22,26,27,30} \\
36-38,42,49-53 \\
65,67,69,72 \\
75,78]\end{array}$ \\
\hline Crambe tataria Sebeók & tátorján & SUB & famine and herdsmen's food & {$[4,14,16,21,55]$} \\
\hline
\end{tabular}




\begin{tabular}{|c|c|c|c|c|}
\hline Scientific name & Local names mentioned & Parts used & Mode of use & Reference No. \\
\hline $\begin{array}{l}\text { Crataegus monogyna Jacq., } \\
\text { Crataegus oxyacantha L. and } \\
\text { related taxa }\end{array}$ & $\begin{array}{l}\text { Istengyümölcs, Istengyümölcsfa, Istengyümöcse, } \\
\text { édeskés galagonya, galaginya, gelegenye, } \\
\text { gyümölcsény, Jézus Krisztus tövise }\end{array}$ & FRU, VEG & $\begin{array}{l}\text { fruits and sometimes leaves were raw } \\
\text { snack; in few places cooked into jam; } \\
\text { dried flour substitute in famine }\end{array}$ & $\begin{array}{l}\text { C. monogyna: } \\
\text { [14,19,22,25, } \\
\text { 27,34,35,39,51, } \\
\text { 59,62,77,78]; } \\
\text { C. oxyacantha: } \\
\text { [22,30,36-38]; } \\
\text { C. sp: }[29,40,46, \\
\text { 50,76] }\end{array}$ \\
\hline Crataegus nigra W. et $\mathrm{K}$. & savanykás galagonya & FRU & raw snack & {$[35]$} \\
\hline $\begin{array}{l}\text { Crocus banaticus L. Gay } \\
\text { (Crociris iridiflorus Heuff.) } \\
\text { \& Crocus balcanicus Janka }\end{array}$ & sáfrány \& balkáni sáfrány & SUB & $\begin{array}{l}\text { spread by wandering herdsmen; bulbs can } \\
\text { be eaten }\end{array}$ & {$[16]$} \\
\hline $\begin{array}{l}\text { Crocus variegatus Hoppe \& } \\
\text { Hornsch }\end{array}$ & vadsáfrány & FLO & spice; flowers were collected and sold & {$[16]$} \\
\hline Dactylis glomerata L. & ebír & VEG & stem was children's snack & {$[78]$} \\
\hline Daphne mezereum L. $\dagger$ & farkasboroszlán & FRU & $\begin{array}{l}\text { unripe fruits were used as a pepper } \\
\text { substitute after cooking }\end{array}$ & {$[4]$} \\
\hline $\begin{array}{l}\text { Daucus carota L. subsp. } \\
\text { carota }\end{array}$ & murok & SUB & added to soups and vegetables & {$[74]$} \\
\hline Echium vulgare L. & édesfü & FLO & $\begin{array}{l}\text { flowers (perhaps fruits also) eaten by } \\
\text { children }\end{array}$ & {$[36]$} \\
\hline Elaeagnus angustifolia $\mathrm{L}$ & olajfa, olajbogyó & FRU & eaten by children & {$[60,78]$} \\
\hline Elymus repens (L.) Gould. & tarackbúza & VEG, SUB & $\begin{array}{l}\text { VEG: raw stem as a snack; SUB: famine } \\
\text { food }\end{array}$ & {$[19,78]$} \\
\hline Equisetum arvense L. & mezei zsurló & FRU & fertile young shoots were eaten by Gypsies & {$[87]$} \\
\hline Eryngium campestre $\mathrm{L}$. & $\begin{array}{l}\text { csipke, bikacsöke, bikacsipke, macskatövis, } \\
\text { széhajtitövis }\end{array}$ & VEG & $\begin{array}{l}\text { young shoots cooked for soup; stems and } \\
\text { fresh leaves were eaten fresh, as salad }\end{array}$ & $\begin{array}{l}{[18,77] \text {; as }} \\
\text { Xanthium } \\
\text { spinosum: } \\
{[59,62]}\end{array}$ \\
\hline Fagus sylvatica L. & bükk, bikfa, májusfa & $\begin{array}{l}\text { FRU, VEG, } \\
\text { SAP }\end{array}$ & $\begin{array}{l}\text { FRU: raw and roasted for snack; for } \\
\text { confectionery as a walnut substitute; } \\
\text { coffee substitute; from seeds oil was } \\
\text { pressed; famine food: ground seeds mixed } \\
\text { into flour (even in 1957); VEG: slightly } \\
\text { acidic leaves eaten raw by children in } \\
\text { the spring, sometimes with sugar; SAP: } \\
\text { children tapped and drank }\end{array}$ & $\begin{array}{l}\text { FRU: }[19,22,23, \\
33,36-39,42,50, \\
\text { 53,57,65,68,69, } \\
72,74,75,78] ; \\
\text { VEG: }[19,36] ; \\
\text { SAP: }[9]\end{array}$ \\
\hline $\begin{array}{l}\text { Fallopia baldschuanica } \\
\text { (Regel) Holub }\end{array}$ & sóskafa, tőtike & VEG & $\begin{array}{l}\text { leaves as a vegetable with a meaty stuffing } \\
\text { filled into them; leaves were used as a } \\
\text { children's snack }\end{array}$ & $\begin{array}{l}\text { [78]; B Czúcz } \\
\text { pers. comm. }\end{array}$ \\
\hline Filipendula vulgaris L. & koloncos legyezőfü, bányavirág & SUB & $\begin{array}{l}\text { sap pressed from roots were eaten; famine } \\
\text { food }\end{array}$ & {$[9,16,21]$} \\
\hline Fragaria moschata Duch. & piroseper, berkeeper, eper & FRU & raw and conserved as jam & {$[75,78]$} \\
\hline Fragaria sp. & $\begin{array}{l}\text { szamóca, bakkeper, fődi eper, földi eper, szimóca, } \\
\text { SK: jahoda }\end{array}$ & FRU & raw and conserved as jam & $\begin{array}{l}{[22,25,26,29,39} \\
42,47,50,52,62 \\
68,76,78]\end{array}$ \\
\hline Fragaria vesca $\mathrm{L}$. & $\begin{array}{l}\text { fődi eper, erdei eper, eper, vereseper, bekeeper, } \\
\text { szamóca, vad eper, piroseper, berekeper, madár- } \\
\text { epörgye, lányeper, bagóeper, erdei szamóca, }\end{array}$ & FRU, VEG & $\begin{array}{l}\text { raw on site; jam, syrup and put into } \\
\text { brandy (palinka) }\end{array}$ & $\begin{array}{l}{[19,27,37,40,49} \\
52,57,72,74,75 \\
77,78]\end{array}$ \\
\hline Fragaria viridis Duch. & $\begin{array}{l}\text { szamóca, csattogó eper, tokoseper, csattogó- } \\
\text { epörgye, lányeper, bagóeper, csattogó, vadeper, } \\
\text { réti szamóca }\end{array}$ & FRU & raw, jam, fruit and liqueur & $\begin{array}{l}{[27,36,37,40,45} \\
53,78]\end{array}$ \\
\hline $\begin{array}{l}\text { Galeobdolon luteum Huds. } \\
\text { (syn. Lamium galeobdolon } \\
\text { (L.) Ehrend \& Polatschek.) }\end{array}$ & $\begin{array}{l}\text { árvacsalyán, árvacsanál, árvalánycsanál, } \\
\text { árvacsihán, szelidcsanál, szopóka }\end{array}$ & FLO & flowers were used as a children's snack & {$[19]$} \\
\hline Galium verum $\mathrm{L}$. & tejoltó galaj & VEG & used for milk inoculation & {$[66]$} \\
\hline $\begin{array}{l}\text { Gentiana cruciata L. \& } \\
\text { Gentiana lutea L. }\end{array}$ & epeburján, epefü, horecska, kösörügyökér, & SUB & put into brandy (palinka), appetizer & {$[19]$} \\
\hline Glechoma hederacea L. & katonapetrezselyem, vadpetrezselyem & VEG & green spice: parsley substitute & {$[19,41]$} \\
\hline
\end{tabular}




\begin{tabular}{|c|c|c|c|c|}
\hline Scientific name & Local names mentioned & Parts used & Mode of use & Reference No. \\
\hline Gleditsia triacanthos $\mathrm{L}$. & $\begin{array}{l}\text { koronatüsökfa, kruskuli, glédicstüsök, vad } \\
\text { szentjánoskenyér, koronaakác, kreditsia, gleditse, } \\
\text { édeske, szejjánoskönyérfa, ledics, lepényfa, } \\
\text { lackószar, zsidótakony, glédicstüsök }\end{array}$ & FRU & $\begin{array}{l}\text { children eat the spongy part of the fruit in } \\
\text { autumn, or suck its sap }\end{array}$ & $\begin{array}{l}{[11,26,27,29,36} \\
38,59,62,63,78]\end{array}$ \\
\hline $\begin{array}{l}\text { Glyceria maxima (Hartm.) } \\
\text { Holm. \& Glyceria fluitans } \\
\text { (L.) R. Br. }\end{array}$ & harmatkása & FRU & flour substitute in famine & {$[16,20,28,60,62]$} \\
\hline $\begin{array}{l}\text { Glycyrrhiza echinata L. \& } \\
\text { Glycyrrhiza glabra L. \& } \\
\text { Glycyrrhiza sp. }\end{array}$ & édesgyökér, idesgyökér & SUB & $\begin{array}{l}\text { sweetener; children's snack; pressed juice } \\
\text { drunk }\end{array}$ & $\begin{array}{l}\text { G. echinata: }[11 \text {, } \\
\text { 22,28,59,62,78]; } \\
\text { G. glabra: }[14, \\
\text { 16,28,57,59,78]; } \\
\text { G. sp.: }[5,60,62]\end{array}$ \\
\hline Helianthus tuberosus L. & $\begin{array}{l}\text { csicsóka, tótrépa, taknyos pityóka, picsóka, } \\
\text { cicoski, mikóka, árvapityóka, árvapijó, } \\
\text { árpapityóka, csókapityóka, csókapicsóka, } \\
\text { disznópityóka, édespityóka, fingópityóka, } \\
\text { fingóspityóka, picsócsa, pityójka }\end{array}$ & SUB, FLO & $\begin{array}{l}\text { stands escaped from cultivation were } \\
\text { gathered; SUB: pickled for winter; } \\
\text { children and adult eat it raw, roasted or } \\
\text { cooked, FLO: nectar was sucked out of } \\
\text { flowers }\end{array}$ & $\begin{array}{l}{[9,43,46,50,52} \\
57,58,62,63,71 \\
74,77,78] ; \text { FLO: } \\
{[52]}\end{array}$ \\
\hline Heracleum sphondylium $\mathrm{L}$. & bojtorján & VEG & $\begin{array}{l}\text { sour soup and refreshing drink was made } \\
\text { from the leaves }\end{array}$ & {$[16]$} \\
\hline $\begin{array}{l}\text { Hippophaë rhamnoides L. } \\
\text { subsp. carpatica Rousi }\end{array}$ & homoktövis & FRU & $\begin{array}{l}\text { whole fruit was conserved in honey in } \\
\text { Transylvania; syrup, jam }\end{array}$ & {$[74]$} \\
\hline Hordeum murinum L. & vadárpa, fü, ragcsos fü, kalász & VEG & stem, spikes were children's snack & {$[78]$} \\
\hline Humulus lupulus L. & komló, vadkomló & VEG, FLO & $\begin{array}{l}\text { FLO: put into bread and beer sourdough } \\
\text { (widespread); VEG: shoots fried (mainly } \\
\text { in Transylvania); soup and vegetable like } \\
\text { French beans }\end{array}$ & $\begin{array}{l}{[7,9,19,27,30,36} \\
38,57,59,67,71 \\
75,77,78,86]\end{array}$ \\
\hline Juglans regia L. & dió, vad dió, dijófa & FRU, SAP & $\begin{array}{l}\text { FRU: into sweets, confectionery; green } \\
\text { husks cooked with sugar to make syrup or } \\
\text { tea; from unripe, soft fruits pickles, jam, } \\
\text { sweets and brandy (palinka) was made. } \\
\text { SAP: children tapped; fresh drink }\end{array}$ & $\begin{array}{l}\text { FRU: seeds in } \\
\text { many sources; } \\
\text { soft fruit: [68]; } \\
\text { green husk: [19]; } \\
\text { SAP: [9] }\end{array}$ \\
\hline Juniperus communis L. & $\begin{array}{l}\text { borsika, borsukafenyő, borókafenyő, bucsfenyő, } \\
\text { sillő, süllő, borosán, borosánfenyő }\end{array}$ & FRU & $\begin{array}{l}\text { spice for brandy (palinka), sour cabbage, } \\
\text { ham marinade and pepper substitute for } \\
\text { meat dishes; used for meat smoking: it } \\
\text { gives a pleasant flavour to meat }\end{array}$ & $\begin{array}{l}\text { [19,22,27,36-38, } \\
\text { 50,52,68,69, } \\
\text { 74,75]; meat } \\
\text { smoking: [27] }\end{array}$ \\
\hline $\begin{array}{l}\text { Koelrauteria paniculata } \\
\text { Laxm. }\end{array}$ & n.d. & FRU & children's snack & {$[78]$} \\
\hline $\begin{array}{l}\text { Lamium album L. \& L. } \\
\text { maculatum (L.) L. \& L. } \\
\text { purpureum L. }\end{array}$ & $\begin{array}{l}\text { árvacsalán, árvacsalyán, árvacsanál, } \\
\text { árvalánycsanál, árvacsihán, szelidcsanál, szopóka }\end{array}$ & FLO & children's snack & {$[19,70,78]$} \\
\hline Larix decidua Mill. & fenyő & SAPs & gum was chewed for cleaning teeth & {$[72,78]$} \\
\hline Lathyrus aphaca L. $\dagger$ & $\begin{array}{l}\text { csiriborsó, csicsiriborsó, feketeborsó, fényesborsó, } \\
\text { finyesborsó, vadborsó }\end{array}$ & FRU & $\begin{array}{l}\text { nice shiny seeds were used for decorating } \\
\text { confectionery }\end{array}$ & {$[9]$} \\
\hline Lathyrus tuberosus L. & $\begin{array}{l}\text { borsó viloja, borsó viola, vadborsó, csunya, } \\
\text { csuma, csunyavirág, julisztavirág, jurisztavirág, } \\
\text { zsírgaz, földimogyoró, fődimagyaró, } \\
\text { földimagyaru, földi zsír, kutyulló fije, borsój, } \\
\text { borsó, borsóvirág, dobra, csicsiriborsó, } \\
\text { csicseriborsó, barabój, jenyestye, unalomvirág, } \\
\text { vadborsóvirág }\end{array}$ & SUB, FRU & $\begin{array}{l}\text { SUB: children and adults ate it almost } \\
\text { everywhere; at ploughing it was ploughed } \\
\text { out or was dug out by pigs; roasted at open } \\
\text { fire (delicious); it has disappeared when } \\
\text { deep ploughing came into practice and } \\
\text { because of herbicides; FRU: seeds were } \\
\text { eaten less often }\end{array}$ & $\begin{array}{l}{[9,11,14,22,26} \\
27,35,49,52,59 \\
60,62,65,67,74 \\
75,77,78]\end{array}$ \\
\hline Lemna sp.* & fulencse & VEG & eaten by poor people & {$[33]$} \\
\hline $\begin{array}{l}\text { Lepidium perfoliatum L. \& } \\
\text { L. ruderale L. }\end{array}$ & borsika, cigánypaprika & VEG & $\begin{array}{l}\text { eaten by Gypsies instead of horseradish } \\
\text { and hot peppers }\end{array}$ & {$[11]$} \\
\hline Lilium martagon L. & n.d. & SUB & $\begin{array}{l}\text { bulbs were put into wine to make vinegar; } \\
\text { herdsmen }>\text { s children ate it dried or was } \\
\text { ground into flour }\end{array}$ & {$[4,65]$} \\
\hline Linaria vulgaris Mill.* & sarkantyúvirág & FLO & flowers were snack & [59] \\
\hline
\end{tabular}




\begin{tabular}{|c|c|c|c|c|}
\hline Scientific name & Local names mentioned & Parts used & Mode of use & Reference No. \\
\hline Lolium perenne $\mathrm{L}$. & fü & VEG & stem was children's snack & {$[78]$} \\
\hline $\begin{array}{l}\text { Lotus corniculatus and/or } \\
\text { L. glaber }\end{array}$ & mogyoró, földimogyoró & SUB & roots (nodules) were children's snack & [78] \\
\hline Lycium barbarum L. $\dagger$ & licium, kutyafa & FRU, FLO & $\begin{array}{l}\text { eaten by children, but in some places } \\
\text { forbidden because it was known as } \\
\text { poisonous; FLO: pistil from the flowers } \\
\text { were children's snack }\end{array}$ & {$[19,62,78]$} \\
\hline Malus sylvestris (L.) Mill. & $\begin{array}{l}\text { vadalma, vadóma, vadóka, madárbogyó, SK: } \\
\text { plánka }\end{array}$ & FRU & $\begin{array}{l}\text { generally everywhere vinegar was made } \\
\text { from it; it was post-ripened, rarely eaten } \\
\text { raw; it was dried; compote, wine, brandy } \\
\text { (palinka) and "cibere" soup was made of } \\
\text { it; as a spice it was put into sour cabbage }\end{array}$ & $\begin{array}{l}{[19,22,23,26,27,} \\
29,36,38-40,42, \\
43,47-53,57-59, \\
62,63,67-69, \\
72,74,76,78]\end{array}$ \\
\hline $\begin{array}{l}\text { Malva neglecta Wallr. \& } \\
\text { Malva sylvestris L. \& Malva } \\
\text { sp. }\end{array}$ & $\begin{array}{l}\text { kalácska virág, papsajt, papsajtmályva, } \\
\text { papsajtja, taknyozófü, kerekmályva, papkalács, } \\
\text { papkenyérke, vadmályva, papsajtlevél }\end{array}$ & FRU & $\begin{array}{l}\text { immature fruit was and is eaten raw by } \\
\text { children }\end{array}$ & $\begin{array}{l}{[9,11,14,21,22} \\
30,42,50,51,59 \\
60,62,70,75 \\
77,78]\end{array}$ \\
\hline $\begin{array}{l}\text { Matricaria recutita L. (M. } \\
\text { chamomilla L.) }\end{array}$ & libavirág, szíkfüvirág, szíkfü, kamilla & FLO & $\begin{array}{l}\text { refreshing tea and medicinal infusion; } \\
\text { flowers were children's snacks }\end{array}$ & {$[29,43,57,78]$} \\
\hline Medicago sativa $\mathrm{L}$. & lucwrna, lucerna & VEG & soup was made from the young shoots & {$[77]$} \\
\hline Melissa officinalis L. & citromfü, citromszagú méhfü & VEG & $\begin{array}{l}\text { modes of use not documented; today } \\
\text { used as a tea and a spice, the plant occurs } \\
\text { spontaneously }\end{array}$ & {$[9]$} \\
\hline Melittis melissophyllum L.* & mecsekháti tea, mecseki-téja, & VEG & $\begin{array}{l}\text { "most popular herb tea"; gathered } \\
\text { basketful for sale; it was made into candies }\end{array}$ & {$[27,29]$} \\
\hline $\begin{array}{l}\text { Mentha sp., M. aquatica L., } \\
\text { M. arvensis L., M. spicata } \\
\text { cv. crispa, M. longifolia (L.) }\end{array}$ & $\begin{array}{l}\text { balzsamka, vad fodormenta, fodorminta, menta, } \\
\text { vízimenta, szagos menta }\end{array}$ & VEG & spice for brandy (palinka); tea & $\begin{array}{l}{[7,11,19,43,67} \\
70,74,78]\end{array}$ \\
\hline Nath., M. pulegium L. & & & & \\
\hline $\begin{array}{l}\text { Morus sp., M. alba L. }(\& M . \\
\text { nigra L.? = M. alba f. nigra })\end{array}$ & $\begin{array}{l}\text { epörgyefa, epörgye, szederfa, faszeder, eperfa, } \\
\text { fekete eper, fehér eper, eperfa, eper }\end{array}$ & FRU & $\begin{array}{l}\text { eaten raw by travellers; brandy (palinka), } \\
\text { wine, jam, syrup made of it, without sugar }\end{array}$ & $\begin{array}{l}{[19,26,27,29,30,} \\
63,78]\end{array}$ \\
\hline Muscari botryoides (L.) Mill. & Szent-györgy virág & VEG, FLO & whole plant was a children's snack & [78] \\
\hline Onopordum acanthium L. & bogáncs & VEG & leaves were a children's snack & {$[78]$} \\
\hline Origanum vulgare $\mathrm{L}$. & ezerjófü, lebetka, lebetkevirág, szúrfü, szurokfü & VEG, FLO & tea; appetizer & {$[19,73,74]$} \\
\hline Oxalis acetosella L. & $\begin{array}{l}\text { medvesósdi, madársósdi, medvesóska, erdei } \\
\text { sósdi, bikksóska, kereksósnya, nyúlsaláta, } \\
\text { nyúlsóska, nyúlsósnya, nyúlsózsnya }\end{array}$ & VEG, FLO & $\begin{array}{l}\text { pressed juice was used like vinegar; } \\
\text { chewed; raw leaves were children's snack; } \\
\text { milk rennet }\end{array}$ & $\begin{array}{l}{[9,16,19,38,70} \\
74,75,78]\end{array}$ \\
\hline $\begin{array}{l}\text { Oxalis corniculata L. (O. } \\
\text { europaea Jord.) }\end{array}$ & $\begin{array}{l}\text { galambsóska, sárga madársóska, kakukksóska, } \\
\text { madársóska }\end{array}$ & VEG & raw snack & [19] \\
\hline Padus avium Mill. & zelnica, szelence, vadszelence & FRU & $\begin{array}{l}\text { eaten by children; put into brandy } \\
\text { (palinka) }\end{array}$ & {$[22,67,74]$} \\
\hline Papaver rhoeas L. & pipacs & FLO & petals were a children's snack & {$[78]$} \\
\hline $\begin{array}{l}\text { Parthenocissus tricuspidata } \\
\text { (S. et Z.) Planch. }{ }^{*} \dagger\end{array}$ & vadszőllö & VEG, FRU & $\begin{array}{l}\text { fruits and thin shoots were put into } \\
\text { pickled cucumbers (even though fruits are } \\
\text { considered toxic) }\end{array}$ & {$[27]$} \\
\hline Pastinaca sativa $\mathrm{L}$. & peszternák, vad pasztinák & SUB & added to soups, children's snack & {$[74,78]$} \\
\hline $\begin{array}{l}\text { Phragmites australis (Cav.) } \\
\text { Steud. }\end{array}$ & nád & SUB, VEG & $\begin{array}{l}\text { SUB: cooked rhizomes are herdsmen's } \\
\text { and famine food; VEG: salad made from } \\
\text { fresh shoots, inner part of the shoots was } \\
\text { spring-summer snack }\end{array}$ & {$[11,14,23,28,78]$} \\
\hline Phytolacca americana L. $\dagger$ & alkörmös & FRU & jam; food colouring & {$[19]$} \\
\hline Picea abies (L.) Karsten & $\begin{array}{l}\text { lucsika, vörösfenyő, veresfenyő, szëmërke } \\
\text { szëmërcefenyő, havasifenyő, csetenyefa, } \\
\text { lukszfenyő, parasztfenyő }\end{array}$ & $\begin{array}{l}\text { VEG, FLO, } \\
\text { SAPs }\end{array}$ & $\begin{array}{l}\text { VEG, FLO: jam, syrup and preserve made } \\
\text { from buds and juvenile cones; SAPs: } \\
\text { gums were teeth cleaner, chewing gum } \\
\text { substitute }\end{array}$ & $\begin{array}{l}{[19,67,70,74} \\
75,78]\end{array}$ \\
\hline Pimpinella anisum $\mathrm{L}$. & ánizs & FRU & $\begin{array}{l}\text { spice for brandy (palinka) and } \\
\text { confectionery }\end{array}$ & {$[77]$} \\
\hline Pinus cembra $\mathrm{L}$. & cirbolya & VEG, FLO & $\begin{array}{l}\text { use not documented; presumably like } P \text {. } \\
\text { sylvestris. Seeds: children snack }\end{array}$ & {$[16,78]$} \\
\hline
\end{tabular}




\begin{tabular}{|c|c|c|c|c|}
\hline Scientific name & Local names mentioned & Parts used & Mode of use & Reference No. \\
\hline Pinus sylvestris L. & $\begin{array}{l}\text { lucfenyő, lucsfenyő, csëmëtefa, csetnye, lúcfenyő, } \\
\text { lukszfenyő, veresfenyö, vörösfenyő, répafenyő }\end{array}$ & VEG, FLO & $\begin{array}{l}\text { syrup was made from young buds and } \\
\text { cones }\end{array}$ & $\begin{array}{l}{[9,19,50,67} \\
69,78]\end{array}$ \\
\hline Plantago lanceolata $\mathrm{L}$. & úti lapi, keskenylevelű útilapi & VEG & preservative was cooked from it; snack & {$[74,78]$} \\
\hline $\begin{array}{l}\text { Plantago major L. \& } \\
\text { Plantago media L. }\end{array}$ & úrfilapi, útilapi, útifü & VEG & raw plant put into butter against anaemia & {$[67]$} \\
\hline $\begin{array}{l}\text { Poa angustifolia L. \& Poa } \\
\text { pratensis L. \& Poa spp. }\end{array}$ & fü & VEG & $\begin{array}{l}\text { stems were used as a children's snack } \\
\text { (chew for the sweet sap) }\end{array}$ & {$[78]$} \\
\hline $\begin{array}{l}\text { Polygonum lapathifolium } \mathrm{L} . \\
\text { \& P. aviculare } \mathrm{L} .\end{array}$ & savanyú keserüfü, lapulevelü keserüfü & VEG & slightly sour leaves were used as snacks & $\begin{array}{l}\text { P. lapathifolium: } \\
{[9,22,57,59] ; P .} \\
\text { aviculare: }[78]\end{array}$ \\
\hline Polypodium vulgare $\mathrm{L}$. & kőméz, édesgyükér & SUB & $\begin{array}{l}\text { sweetener fresh and dried; snack; lapped } \\
\text { into a rag it was given to babies to chew } \\
\text { at teething; "sweet water" was made by } \\
\text { soaking ground roots in water }\end{array}$ & $\begin{array}{l}{[38,42,49,50,52} \\
67,70,74,75,78]\end{array}$ \\
\hline Portulaca oleracea L. & porcsin & VEG, FRU & $\begin{array}{l}\text { eaten; leaves were children's snack; seeds } \\
\text { were famine food }\end{array}$ & {$[16,78]$} \\
\hline Potentilla anserina $\mathrm{L}$. & földi mogyoró, libapimpó & VEG & young leaves were eaten & {$[57,58,62]$} \\
\hline Primula veris $\mathrm{L}$. & kukukvirág & FLO & flowers were cooked into a sweet syrup & {$[75]$} \\
\hline Primula vulgaris Huds. & zsibavirág & VEG & whole plant was consumed & {$[78]$} \\
\hline $\begin{array}{l}\text { Prunus sp. \& P. cerasifera } \\
\text { Ehrh. \& P. domestica L. } \\
\text { subsp. insititia }\end{array}$ & $\begin{array}{l}\text { fosóka szilva, fosóka, kökényszilva, korkodus, } \\
\text { márabora, ringló, vadszilva, macskaszemű szilva }\end{array}$ & $\begin{array}{l}\text { FRU } \\
\text { SAPs }\end{array}$ & $\begin{array}{l}\text { brandy (palinka), preserves, syrup, jam } \\
\text { was made from the fruit; also dried; SAPs: } \\
\text { gum chewing }\end{array}$ & {$[50,68,77,78]$} \\
\hline $\begin{array}{l}\text { Prunus spinosa L. \& } P \text {. } \\
\text { spinosa L. subsp. fruticans } \\
\text { (Weihe) R. et Cam. }\end{array}$ & $\begin{array}{l}\text { kökin, kökényszilva, kükény, kükényszilva, } \\
\text { kökörcsönszilva, kükürcsönszilva, kökén, kükén. } \\
\text { kükénfa, kökönye, porumbar }\end{array}$ & FRU & $\begin{array}{l}\text { eaten mainly raw "after frost-bitten"; wine, } \\
\text { soaked "kökényvíz" (blackthorn water); } \\
\text { brandy (palinka) was made of it; also } \\
\text { dried }\end{array}$ & $\begin{array}{l}{[5,9,11,19,22,23,} \\
25,29,30,36-40, \\
46-48,50-53,57, \\
59,60,62,63,65, \\
70,72,74,76-78]\end{array}$ \\
\hline $\begin{array}{l}\text { Pulmonaria mollis Wulf \& } \\
\text { Pulmonaria officinalis L. }\end{array}$ & dungóvirág, dungófü, tüdőfü & FLO & children suck the nectar out of the flowers & {$[67,70]$} \\
\hline $\begin{array}{l}\text { Pyrus pyraster Burgsd. } \\
\text { (Pyrus achras Gaertn.) }\end{array}$ & $\begin{array}{l}\text { vadkörte, vackor, erdei vackor, vadvackor, malina, } \\
\text { SK: cernice, dicka }\end{array}$ & FRU & $\begin{array}{l}\text { harvested raw, ripened in the attic; stewed } \\
\text { fruit, wine ("csügör"), vinegar, brandy } \\
\text { (palinka) made of it; dried; soup was made } \\
\text { from the dried fruit ("cibereleves") }\end{array}$ & $\begin{array}{l}{[11,19,22,23} \\
25-27,29,36 \\
38-40,42,43 \\
46-53,57-59 \\
62,63,65,68,69 \\
72,74,76-78]\end{array}$ \\
\hline Quercus cerris L. & cserelfa, cserfa, cser & $\begin{array}{l}\text { VEG, SAP, } \\
\text { FRU }\end{array}$ & $\begin{array}{l}\text { VEG: leaves were put into barrelled } \\
\text { cucumber as a spice; SAP: herdsmen } \\
\text { tapped it from spring to late autumn, } \\
\text { "brown beer" for them; FRU: coffee } \\
\text { substitute after leaching and roasting, } \\
\text { famine food }\end{array}$ & $\begin{array}{l}\text { SAP: }[24,27,28 \\
\text { 38,39,44]; VEG: } \\
{[19,77] ; \text { FRU: }} \\
{[16]}\end{array}$ \\
\hline Quercus pubescens Willd. & magyal & FRU, VEG & $\begin{array}{l}\text { FRU: herdsmen roasted the acorns } \\
\text { on ember; VEG: leaves were put into } \\
\text { barrelled cucumber as a spice }\end{array}$ & {$[14,16,77]$} \\
\hline $\begin{array}{l}\text { Quercus robur L. \& Q. rubra } \\
\text { L. \& Q. petraea (Matt.) }\end{array}$ & cserefa, tölgy, tölgyfa, töfa, tőfa, töljfa csepefa & VEG, FRU & $\begin{array}{l}\text { VEG: leaves were put into barrelled } \\
\text { cucumber as a spice; bark: famine flour }\end{array}$ & $\begin{array}{l}\text { VEG: [77]; FRU: } \\
{[19,23,27,33,35}\end{array}$ \\
\hline Liebl. \& Quercus sp. & & & $\begin{array}{l}\text { substitute FRU: coffee substitute, children's } \\
\text { snack and famine flour substitute: boiled } \\
\text { acorns lost their bitter taste, after boiling it } \\
\text { was dried, ground and mixed into flour }\end{array}$ & $\begin{array}{l}\text { 37,48,77,78]; } \\
\text { Bark: }[14]\end{array}$ \\
\hline Ranunculus ficaria L. & $\begin{array}{l}\text { györgysaláta, papsaláta, szentgyörgybúza, } \\
\text { madársaláta, galambsaláta, vadsaláta, búzasaláta, } \\
\text { bükki saláta, erdejisaláta, harangversengő, } \\
\text { kereksajáta, kakuksaláta, kukuksaláta, } \\
\text { kukuksajáta, mezejisaláta, nyúlsaláta, nyúlsajáta, } \\
\text { salátavirág, szaronkőtt saláta, vadsajáta, } \\
\text { pipirisaláta }\end{array}$ & VEG & $\begin{array}{l}\text { it was consumed mainly raw, with a sauce } \\
\text { as a spring salad; roasted in speck lard } \\
\text { with garlic; cooked for soup and vegetable } \\
\text { (tradition survived in Transylvania); } \\
\text { in Hungary it re-appears in markets, } \\
\text { gathered by Gypsies }\end{array}$ & $\begin{array}{l}{[9,27,31,35,58} \\
62,65,70,71,74 \\
77,78]\end{array}$ \\
\hline Ribes alpinum L. & leánykafüge, vad ribizli & FRU & eaten raw, wine was made from it & {$[70,75]$} \\
\hline
\end{tabular}




\begin{tabular}{|c|c|}
\hline Scientific name & Local names mentioned \\
\hline Ribes aureum Pursch & aranyribizli \\
\hline Ribes nigrum $\mathrm{L}$. & $\begin{array}{l}\text { fekete ribizli, fekete ribizli, fekete szöllő, fekete } \\
\text { vërësszőlő }\end{array}$ \\
\hline Ribes petraeum Wulf. & borfüge \\
\hline Ribes rubrum L. & piros ribizli, vad ribizli \\
\hline Ribes uva-crispa L. & $\begin{array}{l}\text { egres, egris, füge, szőrős füge, vad egres, agris, } \\
\text { piszke, büszke }\end{array}$ \\
\hline Robinia pseudacacia L. & $\begin{array}{l}\text { akác, fehér akác, agacsi, ágác, mézvirág, kukucka, } \\
\text { agáca }\end{array}$ \\
\hline $\begin{array}{l}\text { Rosa canina L. agg. \& Rosa } \\
\text { corymbifera Borkh. (Rosa } \\
\text { dumetorum Thuill.) \& Rosa } \\
\text { sp. }\end{array}$ & $\begin{array}{l}\text { csipkerózsa, hecseli, hecsedli, hecserli, } \\
\text { rózsabogyó, pecs, bucske, bücske, bütyke, } \\
\text { seggvakaró, seggvakarcs, istengyümőcs, vadrózsa, } \\
\text { csipkefa, szaragógya, szagrógya, bicskerózsa, } \\
\text { csitkenye, csitke, csipka, hecse, hecse-pecse, } \\
\text { hecsebokor, tüvisfa hecsempecs, csicskenye, } \\
\text { csipkebokor }\end{array}$ \\
\hline
\end{tabular}

Rosa gallica L.

Rubus caesius L. \& Rubus sp. szeder, seder, födi szeder, erdei szeder, födi (also as Rubus tomentosus szödörnye, szödör, csuszkor, promber, futó Borkh)

Rubus fruticosus agg.

Rubus idaeus L.

Rumex acetosa L. \& Rumex sp.

Rumex acetosella L.

\section{Rumex patientia L.}

Rumex pseudonatronatus

Borb.*

Sagittaria sagittifolia L. Salvia pratensis L. \& S. nemorosa $\mathrm{L}$. lószamóca

málna, mána, malina, mánafa, málnafa vadsóska, papsaláta, sósdi, tavaszi sóska, sóski, sósnya, vadsósnya, lósóska

Parts used

Mode of use

Reference No.

\begin{tabular}{|c|c|c|}
\hline FRU & snack & {$[78]$} \\
\hline RU & eaten raw (also in gardens) & {$[9,50,74]$} \\
\hline RU & eaten raw & {$[75]$} \\
\hline $\mathrm{U}$ & eaten raw (also in gardens) & {$[38,74]$} \\
\hline $\mathrm{U}$ & eaten raw; gathered for preserves and soup & $\begin{array}{l}{[9,38,70,74} \\
75,78]\end{array}$ \\
\hline \multirow[t]{5}{*}{ FLO, VEG } & FLO: whole flower or just the nectar was & FLO: $[19,23,27$, \\
\hline & children's snack; in many places it was & $29,31,36,38,50$ \\
\hline & fried in pancake dough; tea; less often & $59,60,70,74,77$ \\
\hline & wine was made of it; sometimes added to & 78]; to bread: \\
\hline & bread sourdough. VEG: young leaves were & [17]; VEG: [59] \\
\hline
\end{tabular}

FRU, FLO FRU: syrup, jam, preserves, stewed fruit, soaked drink; with yeast it was made into wine; "cibere" soup, enriched with bread; dried: tea; ground: flour substitute in famine; FLO: refreshing drink, vinegar and preserves were made from petals

R. canina: $[9,11$, $19,22,25,26,29$, $30,36-40,42,43$, $45-50,52,53,57$, $60,65,68,71$, 74-78]; $R$. corymbifera: [19, 22]; Rosa sp.: $[22,59,62,74]$

FRU, FLO, FLO: syrup and jam were made from the $[19,22,23,74,76]$ VEG petals with sugar and citric acid. VEG: tea from leaves. FRU: tea, jam, syrup.

FRU, VEG FRU: eaten raw in the past and also today; R. caesius: [9,11, jam, preserves, wine, brandy (palinka), 19,22,26,27,30, syrup, thick jam ("dulcsesz") was made of $35,36,45,47,49$, it. VEG: tea from leaves 52,72,74,76-78]; Rubus sp.: $[22,25,29,30,36$, $38,42,43,48,52$, $57-60,62,68]$

szeder, fekete szeder, fás szödörnye, tüskeszeder, FRU

FRU, VEG

sóska, réti sóska, vadsóska, sóslorjum, sóslórum, VEG

FRU: eaten raw in the past and also today; wine, brandy (palinka) was made of it; sold in markets

$[19,22,27,38,42$, $65,75,77,78]$

$[19,23,38,40,42$, $43,45,47-52,65$, (palinka) was made of it; also put into $\begin{array}{ll}\text { brandy (palinka); fruits were conserved in } & 67,70,72, \\ \text { syrup. VEG: tea was made from leaves. } & 74-76,78]\end{array}$ mentioned by almost all sources. It was an R. acetosa: $[9,19$, important spring vegetable everywhere; $\quad 22,23,27,35,36$, field snack, eaten raw mainly by children; $\quad 38,39,42,43,46$, cooked for soup and sauce; also used for $\quad 49-53,57-59,62$, inoculation of milk

65-67,70,77,78];

Rumex sp.: [33, $47,60,71,78]$

mainly snack; sometimes eaten after $[43,49,50,52,65$, $66,78]$ inoculation of milk cooked for sauce and soup

$[5,31,43,76,78]$ [59]

$[14,16]$

alacs, elecs, elecske

SUB

famine food

$[75,78]$ 
Tab. 1 (continued)

$\begin{array}{llll}\text { Scientific name } & \text { Local names mentioned } & \text { Parts used } & \text { Mode of use }\end{array}$

Sambucus ebulus L. $\dagger$

fődi bodza, földi bodza, gyalogbodza, gyalog bédza, födi-boza, borzang, fekete borzag, csete

FRU

FLO

Sambucus nigra L. \& Sambucus sp.

fabodza, borza, bodzavirág, gyalogbodza, bodza, fehér bozza, bozda, bozzafa, bojzafa, fekete bojza, bodzafa, bozdafa, borzag, borza, borzafa

n.d.

n.d.

fekete gyökér

bakceka

muhar

repce, rabcsont, rebcsont

magas zsombor

Sisymbrium altissimum $\mathrm{L}$.

(as Sisymbrium pannonicum

Jacq.)

Smyrnium perfoliatum L.

Solanum nigrum L. $\dagger$

őzsaláta

fekete szöllő, fekete vad szöllő, káposztaszöllő

VEG

FRU

\section{Solidago gigantea Ait.}

Sonchus arvensis L.

Sorbus aucuparia L.

Sorbus domestica L. \&

Sorbus sp.

Sorbus torminalis (L.) Cr.

Staphylea pinnata L.*

Stellaria media (L.) Vill.

Streptopus amplexifolius

(L.) DC

Symphytum officinale L. $\dagger$

Taraxacum officinale Weber

\& T. laevigatum (Willd.)

DC.

Taxus baccata L. $\dagger$

Thymus serpyllum L. \&

Thymus sp.

Tilia platyphyllos Scop. \&

Tilia cordata Mill. \& Tilia

sp.

SUB

VEG

vadkender

VEG

belekenyér, istenkenyere

berkenye, berkenyi

FRU

klokocs,

galambbegy, tyúkhúr, korpafü

FRU

VEG

nyúleper

feketenadály, fekete nadálytő, dongóvirág tyúkvirág, pitypang, pipevirág, marcivirág, láncfü, kákics, kutyavirág, pimpó, békavirág, bíkavirág, cikornya, csikāra, hóttok virága, kotlóvirág, láncoslapu, nyúlsaláta, pipefü, pipevirág, tejesbúrjány, tejesbúrján, tejesfü, tejefü, fügörhe, tejesgaz, tejes vadsaláta, tyúksegge, csorbóka, libuskavirág

\section{tiszafa}

FRU

csombor, kakukkbora, kakukkfüj, vadborsfüj, vadcsombor

hársfa
FRU: jam and syrup was cooked, brandy (palinka) was distilled from it; jam was made mixed with pumpkins. It was not eaten raw, and was used less often than $S$. nigra. FLO: tea.

FLO, FRU FRU: jam was cooked; brandy (palinka) was distilled from it; used for colouring wine. FLO: tea, refreshing drink, syrup, "elder champagne"; fried in pancake dough: "elder doughnut"

FRU red fruits were eaten

VEG mountain herdsmen used it as a spice

eaten

children's snack

FRU: [19,26,27,

$30,37-39,45,69$

77]; FLO: [77]

CZB

$[5,9,19,27,30,37$, $42,43,49,50,52$,

$57,59,60,62,67$, $69,74,75,78]$

[36,37]

stem was used as a children's snack (chewed for the sweet sap)

VEG, FRU FRU: oil pressed; VEG: soup was made from young spring shoots

FRU oil pressed

$[4,7476,77]$

salad was made from raw leaves sweet fruits were eaten by children (it is considered poisonous, but the source notes: "1-2 pieces do not make any harm")

children's snack

[78]

n.d.

[65]

fruits eaten

$[19,69]$

FRU dried

$[22,28,36,37]$

FRU sweet, pulpy fruits were eaten by children, [37-40] herdsmen

eaten by children

[38]

eaten as salad and vegetable

$[11,78]$

FRU fruits ripen in hay by winter, eaten raw

VEG, FLO VEG: young leaves were eaten fried in harsh times; FLO: children's snack

FLO: [9]; VEG:

[14], SZGY

$[9,19,22,28,50$,

$59,63,65,70,74$, 75,78]; $T$.

VEG: young leaves were eaten as salad in spring; FLO: syrup was cooked from soaked flowers; or "cikoria honey" was made from flowers with thick sugar syrup; laevigatum: in Gömör "pimpó wine" was fermented $[59,62]$ from the flowers; in some places it was regarded as poisonous

arils were eaten as children's snack spice

$[19,30,60,62,78]$

tea; condiment for brandy (palinka) and wine
$[19,27,29,38,40$, $43,61,78]$ 


\begin{tabular}{|c|c|c|c|c|}
\hline Scientific name & Local names mentioned & Parts used & Mode of use & Reference No. \\
\hline $\begin{array}{l}\text { Tragopogon orientalis L. (as } \\
\text { Tragopogon pratensis L.) }\end{array}$ & $\begin{array}{l}\text { bakceka, bakszaka, baszkata, csuka, édesfü, } \\
\text { idesfü, tefesfü, kukukté, szasza, szekeboka, } \\
\text { tejesbúrján, tejesbúrjány }\end{array}$ & VEG & $\begin{array}{l}\text { in the past young sweet stems were eaten } \\
\text { peeled; in autumn curly leaves were } \\
\text { chewed; in some places it was cooked in } \\
\text { whey }\end{array}$ & {$[9,50,65,67,75]$} \\
\hline Trapa natans $\mathrm{L}$. & sulyom, suly, sójom & FRU & $\begin{array}{l}\text { cooked and roasted; flour substitute in } \\
\text { famine; till the 1930s it was sold in large } \\
\text { quantities }\end{array}$ & $\begin{array}{l}{[5,11,14,16,30} \\
33-35,58-60,62]\end{array}$ \\
\hline $\begin{array}{l}\text { Trifolium pannonicum } \\
\text { JACQ }\end{array}$ & nagy fehér vad here & FLO & $\begin{array}{l}\text { syrup was cooked from the flowers; } \\
\text { children's snack }\end{array}$ & {$[74]$} \\
\hline Trifolium pratense $\mathrm{L}$. & vörös lóhere, lucerna, istenke cipókája & FLO & children's snack & {$[16,35,78]$} \\
\hline Trifolium repens $\mathrm{L}$. & lóhere & VEG & $\begin{array}{l}\text { leaves and stem were used as a children's } \\
\text { snack }\end{array}$ & {$[78]$} \\
\hline $\begin{array}{l}\text { Tripleurospermum } \\
\text { perforatum (Mérat) M. } \\
\text { Lainz }\end{array}$ & kamilla & FLO & flowers were children's snacks & {$[78]$} \\
\hline Tussilago farfara L. $\dagger$ & $\begin{array}{l}\text { fodbájlapi, podbánlapi, podbállapi, martilapi, } \\
\text { martilapu, martivirág, partilapu, pipevirág, } \\
\text { ciberelapu, fehérhátu lapu, tejfölös lapu, pitypang, } \\
\text { gyermekláncfü }\end{array}$ & VEG, FLO & $\begin{array}{l}\text { VEG: stuffed with meat or mush; soup, } \\
\text { vegetable, salad, in spring salad soup with } \\
\text { bones (first cooking water was poured } \\
\text { off, because it was bitter); FLO: flowers } \\
\text { cooked into sugar syrup }\end{array}$ & $\begin{array}{l}{[9,19,65,67} \\
71,74]\end{array}$ \\
\hline $\begin{array}{l}\text { Typha latifolia L. \& Typha } \\
\text { angustifolia L. }\end{array}$ & $\begin{array}{l}\text { csella, elecske, bengyele, pintér gyékény, nádi } \\
\text { botikó }\end{array}$ & SUB, VEG & $\begin{array}{l}\text { SUB: rhizomes were eaten raw or cooked } \\
\text { by herdsmen; famine food; VEG: salad } \\
\text { from young leaves }\end{array}$ & $\begin{array}{l}{[4,14,28,30,34} \\
59,61,62,78]\end{array}$ \\
\hline $\begin{array}{l}\text { Ulmus glabra Huds. ?=? } \\
\text { Ulmus minor Mill. \& Ulmus } \\
\text { sp. = U. laevis L.* }\end{array}$ & szilfa & SAP & fresh drink & {$[27,33]$} \\
\hline $\begin{array}{l}\text { Urtica sp., Urtica dioica L. \& } \\
\text { Urtica urens L. }\end{array}$ & $\begin{array}{l}\text { csijány, csián, csiján, csalán, csihán, csallán, } \\
\text { csollán, csohán, csonár, csajánt, csojánt, csoján, } \\
\text { csojány, csohány, csípős csajánt, csípős csojánt, } \\
\text { csípős csojány, csípős csollán, széleslevelü csoján, } \\
\text { csípős csohán }\end{array}$ & VEG & $\begin{array}{l}\text { soup, vegetable and salad was made from } \\
\text { fresh shoots; weak children were fed with } \\
\text { nettle; eggs with nettle: eastern dish; tea }\end{array}$ & $\begin{array}{l}{[9,19,23,28,33} \\
36,48,50,52,53 \\
63,65,71,74-78] \\
\text { NI; U. urens: } \\
{[52,53,65,77]}\end{array}$ \\
\hline $\begin{array}{l}\text { Vaccinium } \\
\text { gaultherioides Bigelow }\end{array}$ & takonkokojza & FRU & eaten raw & {$[75]$} \\
\hline Vaccinium myrtillus L. & $\begin{array}{l}\text { feketekukujza, feketekokojza, kakóca, kakojza, } \\
\text { fekete áfonya, fekete kukojsza, áfinya, áfinyála, } \\
\text { háfonya, háfinya, feketemeggy, boronyica, } \\
\text { brusnyica }\end{array}$ & FRU, VEG & $\begin{array}{l}\text { FRU: raw; as jam, liqueur, syrup, } \\
\text { preserves; preserved in rum; put into } \\
\text { brandy (palinka); VEG: tea from leaves }\end{array}$ & $\begin{array}{l}{[9,16,19,30} \\
48-52,70,72 \\
74-76,78]\end{array}$ \\
\hline Vaccinium vitis-idaea $\mathrm{L}$. & $\begin{array}{l}\text { piroskokojza, piroskukujza, fojminc, piros } \\
\text { kukojsza, piros kakojza, havasi meggy, vörös } \\
\text { áfonya, piros áfonya, ménisora, fásmeggy, } \\
\text { botonyica, brusnyica }\end{array}$ & FRU & $\begin{array}{l}\text { eaten raw, fresh and as pickles; } \\
\text { preservation: pickled; sweet preserves; put } \\
\text { into brandy (palinka) and wine; cooked } \\
\text { into wine: glee; preserved in alcohol; } \\
\text { dried; harvested semi-ripe and ripened in } \\
\text { the sun }\end{array}$ & $\begin{array}{l}{[19,48-52,70,74} \\
75,78]\end{array}$ \\
\hline $\begin{array}{l}\text { Valerianella locusta (L.) } \\
\text { Latterade }\end{array}$ & $\begin{array}{l}\text { vadsaláta, nyúlsaláta, galambbegy saláta, madárka } \\
\text { saláta }\end{array}$ & VEG & eaten in spring as salad, poured with sauce & {$[19,27,38,53,78]$} \\
\hline $\begin{array}{l}\text { Valerianella olitoria (L) Poll. } \\
\text { or V. dentata (L.) Poll. }\end{array}$ & madársaláta, papsaláta, vadsaláta & VEG & eaten as salad with oil and vinegar & {$[22]$} \\
\hline Verbena officinalis $\mathrm{L}$. & vasfü, vasfüj, vasfi, szaporagaz & VEG & $\begin{array}{l}\text { put into pickled cucumbers; spice and } \\
\text { preservative }\end{array}$ & {$[9,38,67]$} \\
\hline $\begin{array}{l}\text { Viburnum lantana L. } \dagger \& \\
\text { Viburnum sp. }\end{array}$ & $\begin{array}{l}\text { nyomittó, nyomtató, ostorménfa, barátsza, } \\
\text { barátszar, farkascseresnye, gusfa, bangita, } \\
\text { korbácsnyélfa, ostórminya, ostornyélfa, szentfa, } \\
\text { Szent-Ilona szőlő, szereputyka }\end{array}$ & FRU & fruits and pressed juice; snack for children & {$[9,19,26,67,74]$} \\
\hline Viburnum opulus L. $\dagger$ & $\begin{array}{l}\text { kányafa, kánya, veres kánya, gána, kálenka, } \\
\text { kalina, kalinafa, kalinka }\end{array}$ & FRU & $\begin{array}{l}\text { raw when frost-bitten but bitter: jam and } \\
\text { syrup was made of it }\end{array}$ & {$[19,74]$} \\
\hline Vicia spp. & csicseriborsó, vadborsó & FRU & cakes were decorated with its shiny seeds & [9] \\
\hline $\begin{array}{l}\text { Vitis sylvestris C. C. Gmel. } \\
\text { (V. vinifera L. subsp. }\end{array}$ & vadszőlő & FRU & $\begin{array}{l}\text { children's snack; vinegar and brandy } \\
\text { (palinka) was fermented from it }\end{array}$ & {$[22,27,68,78]$} \\
\hline
\end{tabular}

sylvestris) 
Further poisonous species, tasted and snacked by some Hungarian botanists in their childhood include Chelidonium majus L, VEG Conium maculatum L. (VEG: piece of internode), Hedera helix L. (FRU), Ligustrum vulgare L. (FRU), Parthenocissus quinquefolia (L.) Planch (FRU), Salix alba L. (VEG: leaves), Syringa vulgaris L. (VEG: branch).

FLO - flowers, inflorescences, sometimes only petals or nectar; FRU - fruits or seeds; n.d. - no data about local names in the sources; SAP liquid sap of trees; SAPs - dried forms of saps (resin or gums); SUB - subterranean parts (rhizomes, roots, bulbs, tubers); VEG - leaves, shoots, buds, sometimes the whole plant. ${ }^{*}$ Uncertain identification (species inclusion in the list is based only on ethnographic sources). $\dagger$ Plant (the consumed part) is mentioned to be poisonous by some sources.

maritimus, Butomus umbellatus, Crambe tataria, Filipendula vulgaris, Phragmites australis, Sagittaria sagittifolia and Typha latifolia were known as important famine foods. Crambe tataria and Typha latifolia were reported to be preferred to bread as a staple food by some herdsmen even in normal times [60]. Collecting and eating Lathyrus tuberosus tubers used to be a widespread practice after ploughing. Polypodium vulgare and Glycyrrhiza rhizomes were generally consumed as kids' snacks and used as a sweetener. Tubers of Chaerophyllum bulbosum and Helianthus tuberosus were important wild vegetables eaten raw and cooked. Acorus calamus and Gentiana roots were used as pálinka spices; whereas Cichorium intybus roots can serve as a coffee substitute, still in use Transylvania [74].

\section{Tree saps}

Tapping trees for sap used to be a common practice in the forested regions of Eurasia. Saps from Betula pendula, Quercus cerris, Carpinus betulus, Ulmus spp. and Acer pseudoplatanus trees were generally drunk raw. Carpinus betulus, Fagus sylvatica and Juglans regia trees were also tapped in Transylvania. Betula pendula sap used to be an important commercial item sold in larger quantities at markets as a refreshing beverage, a medicine or a curdling agent. Quercus cerris sap was an important water source for herdsmen where spring water was scarce. Oaks giving the best sap were tapped permanently with built-in elder tubes; some trees were visited for drinking for 20 years. The dried resin of Picea abies, Larix decidua, Prunus cerasifera and Cerasus avium was used for chewing. This was not only useful for cleaning teeth, but also for stimulating the production of saliva, which was needed for spinning.

\section{Social aspects of the gathering activities}

Wild food plants, especially fruits, were collected both for own use or for sale. Unwritten laws regulated gathering. Signing an area rich in blueberries or strawberries, or a wild fruit tree, or arriving first in the gathering day, anyone could reserve the fruits for himself [16]. According to a documented tradition [48], families without horses were allowed to do gathering in closer to the village than families possessing horses. Horses knew their job, they followed their owners with baskets on their backs. Gathering traditions went from generation to generation in communities, sometimes in families. Jam making from Sambucus ebulus was a community activity among German-speaking people till the 1930s, like jam-making from Rosa canina in some villages; this latter tradition exist even today (e.g. in the village Szarvaskő), but more as a tourist attraction.

It was also noted that some people were ashamed that they need to eat green leaves, and were unwilling to speak about it, since gathering ("nettle-eating") was often regarded as a sign of poverty in some communities [9]. Teachers coming from an urban environment also influenced children to give up snacking on wild plants: in a documented case children have to write down 500 times "grass is eaten only by ruminant beasts" as a punishment [50].

\section{Discussion}

The diverse natural vegetation of the Carpathian Basin was a rich source of wild food plants. The consumption of wild plants was of different magnitude and economic significance in different periods and different regions, depending both on the natural characteristics of the landscape and the socio-economic background and tradition-preserving ability of the families or communities. The importance of gathering activities changed in parallel with the areal loss of natural vegetation and with socio-economic changes. The greatest changes occurred in the lowland floodplains, where traditional gathering, fishing and hunting activities provided livelihood for many people. After the extensive drainage of the floodplains in the late 19th and early 20th centuries, the utilization of wild food plants dropped dramatically. The two main reasons behind this transition were the reduced availability of the most important wetland plants and the new economic opportunities in the transformed landscape dominated by arable fields, which rendered gathering unnecessary, and left no time for such activities [16,32,35]. The tradition of consuming wild food plants could survive for a longer time in forested and mountain regions unsuitable for agricultural production, where the proportion of natural vegetation was larger. In such regions there were several documented cases of people actively consuming 20-30 species even in the middle of the 20th century (e.g. [27,36,50,74,75]). Snacking of wild fruits, flowers, and raw wild vegetables survived longest among herdsmen and children.

Today, the gathering and consumption of wild plants is increasingly becoming popular and fashionable activity again. Factors behind this process include health-conscious nutrition as well as the worsening economic situation experienced by many. Some species e.g. Allium ursinum, A. scorodoprasum, Sambucus nigra, Ranunculus ficaria, Rosa canina, Prunus spinosa, Rubus, Crataegus, and Urtica spp., are appearing in markets as sources for fashionable "nature products". Gathering wild plants for sale is generally a seasonal subsistence activity of the poorest people - e.g. gypsies or elderly people. Under harsh economic conditions the consumption of wild goods becomes a matter of survival. There always have been (and there are still) places, where indigent families made use of everything "presented by the Creator in his endless good will since the beginning of times" [63]. 
More traditional forms of gathering activities can also be a living tradition today. Among Hungarians living in Transylvania the traditional use of wild plants have continued till today in many places. There are settlements, where more than 30-40 wild species are used in everyday life [74,75], and wild fruits (e.g. Fragaria, Vaccinium spp.) are commonly sold in markets.

Comparing Hungary to Mediterranean (e.g. Spain and Italy $[1,2])$ and to the countries north of Hungary (Poland $[3,80,81]$, Slovakia [82], Estonia [83]) there are some striking similarities and differences. Use of most wild fruits and seeds, as well as the little importance of wild greens is similar to that of Poland, Slovakia and Estonia. Similar to several Mediterranean countries, the consumption of the members of Liliaceae is high in Hungary.

A specific feature of the traditional plant use of the Hungarians is the mass consumption of the underground parts of some wetland species, particularly Typha spp. but also Phragmites, Sagittaria, Alisma, Butomus, and Bolboschoenus. Another Hungarian characteristic is the widespread consumption of some continental steppe species, reaching the Great Hungarian Plain from the east, most notably Crambe tataria, Rumex pseudonatronatus and the endemic Armoracia macrocarpa. The traditional knowledge of these plants might have arrived into the Carpathian Basin with the Hungarians coming from the Eurasian steppes in 896 AD. The Turkic origin of the common names of several species (e.g. "tátorján": Crambe tatarica, "bojtorján": Arctium lappa, "gyékény": Typha latifolia, "som": Cornus mas, "katáng": Cichorium intybus) suggests that these species might have been consumed before the Hungarians settled in the Carpathian basin. Interestingly, there are no records of Hungarians using some species (e.g. Aegopodium podagraria, Alliaria petiolata, Sonchus oleraceus, Lactuca serriola, Stachys palustris) common in the Carpathian Basin, which are important wild vegetables in other European countries, Poland, Germany, Spain or Italy [1-3,79-84].

\section{Conclusions}

We reviewed 71 papers and manuscripts summarizing information on the use of 236 species. Although we did our best to find all relevant papers, it is still necessary to search for further literature, and, particularly, to perform additional field data collection in order to record the traditions still surviving in some regions. One might ask: is it not too late? Does the knowledge on wild food plants and their traditional use still exist? As a few contemporary field studies testify, there is some hope left. There are places where this kind of traditional knowledge still exists, and even new species or new uses for old species can be documented. There are hints that this knowledge may exist also in other regions, and some communities consciously revitalize ancient traditions for economic or other reasons (e.g. the production of rosehip jam as a community activity in Szarvaskő village). But the erosion of traditional knowledge on wild food plants is very fast.

There are several regions where no research on this topic was ever pursued, (e.g. Hanság, Zselic, Mecsek, Balaton region); and the traditional wild plant use of non-Hungarian ethnic groups living in Hungary (e.g. Germans) would be also worth to explore. Exploring and documenting this form of traditional ecological knowledge is an important part of conserving cultural heritage. Lessons on sustainable interactions between nature and human communities can become an important source of information in an uncertain and energy scarce future [85]. In addition to serving as a basis for novel business opportunities, traditional forms of wild plant use can also improve the cohesion and resilience of local communities.

\section{Acknowledgements}

We are grateful to István Burján and Katalin Sárközi (Ethnography Department of Janus Pannonius Museum, Pécs) for their assistance in tracking down literature sources. We are also grateful to the 32 Hungarian botanists who responded to our query on their childhood snacks: Lajos Balogh, János Bölöni, Anikó Csecserits, Anna Mária Csergő, Ágnes Csomós, Áron József Deák, Tamás Exner, Sándor Farkas, Alexander Fehér, Gábor Fekete, Ferenc Gyulai, Eszter Illyés, Melinda Juhász, Árpád Kenéz, Géza Kósa, András Kun, Imre Majláth, Ákos Malatinszky, András Máté, József Nagy, Miklós Óvári, Róbert Pál, Tamás Pócs, Szilvia Rév, Dénes Saláta, Imelda Somodi, Klára Szabados, István Szabó, László Gyula Szabó, Attila Takács, Tamás Tóth, Gábor Turcsányi.

\section{References}

1. Tardío J, Pardo-De-Santayana M, Morales R. Ethnobotanical review of wild edible plants in Spain. Bot J Linn Soc. 2006;152(1):27-71. http:// dx.doi.org/10.1111/j.1095-8339.2006.00549.x

2. Ghirardini M, Carli M, Del Vecchio N, Rovati A, Cova O, Valigi F, et al. The importance of a taste. A comparative study on wild food plant consumption in twenty-one local communities in Italy. J Ethnobiol Ethnomed. 2007;3(1):22. http://dx.doi.org/10.1186/1746-4269-3-22

3. Łuczaj Ł. Dziko rosnące rośliny jadalne użytkowane w Polsce od połowy XIX w. do czasów współczesnych. Etnobiologia Polska. 2011;1:57-125.

4. Molnár V. A. Kitaibel Pál élete és öröksége. Biatorbágy: Kitaibel Kiadó; 2001.

5. Oláh A. Zöld varázslók, virág-orvosok: népi gyógynövényismeret Békés megyében. Békéscsaba: Békés Megyei Tanács V. B. Tud.-Koordinációs Szakbizottság; 1987.

6. Kóczián G, Pintér I, Gál M, Szabó I, Szabó L. Etnobotanikai adatok Gyimesvölgyéből. Botanikai Közlemények. 1976;63(1):29-35.

7. Kóczián G, Szabó I, Szabó L. Etnobotanikai adatok Kalotaszegről. Botanikai Közlemények. 1977;64(1):23-29.

8. Papp N. Népi gyógynövény-ismereti kutatások a kolostori gyógyászatban és Erdélyben (2007-2010). Journal of History of Culture, Science and Medicine. 2011;2(2):76-88.

9. Péntek J, Szabó A. Ember és növényvilág: kalotaszeg növényzete és népi növényismerete. Bucharest: Kriterion; 1985.

10. Molnár Z, Babai D. Népi növényzetismeret Gyimesben I. Növénynevek, népi taxonómia, az egyéni és közösségi növényismeret. Botanikai Közlemények. 2009;96(1-2):117-143.

11. Molnár Z. A traditional ecological knowledge of herders on the flora and vegetation of the Hortobágy. Debrecen: Hortobágyi Természetvédelmi Közalapítvány; 2012.

12. Fekete G, Varga Z, editors. Magyarország tajainak növényzete és állatvilága. Budapest: MTA Társadalomkutató Központ; 2006.

13. Borhidi A. Magyarország növényföldrajzi képe. A magyar flóra származása és elemei. In: Fekete G, Varga Z, editors. Magyarország tajainak növényzete és állatvilága. Budapest: MTA Társadalomkutató Központ; 2006. p. 27-38.

14. Rapaics R. A kenyér és táplálékot szolgáltató növényeink története. Budapest: Királyi Magyar Természettudományi Társulat; 1934. (Népszerű Természettudományi Könyvtár; vol 16). 
15. Gunda B. A magyar gyüjtögető és zsákmányoló gazdálkodás kutatása. Budapest: Néptudományi Intézet; 1948. (Magyar Népkutatás Kézikönyve; vol 2).

16. Gunda B. A vadnövények gyüjtése. In: Paládi-Kovács A, editor. Magyar néprajz II. Gazdálkodás. Budapest: Akadémiai Kiadó; 2001. p. 11-40.

17. Kisbán E. Táplálkozáskultúra. In: Paládi-Kovács A, editor. Magyar néprajz IV. Anyagi kultúra 3. Életmód. Budapest: Akadémiai Kiadó; 1997. p. 417-584.

18. Morvay J. Népi táplálkozás. Budapest: Magyar Nemzeti Múzeum; 1962. (Útmutató füzetek a néprajzi adatgyüjtéshez).

19. Kóczián G. A hagyományos parasztgazdálkodás termesztett, a gyüjtögető gazdálkodás vad növényfajainak etnobotanikai értékelése [ $\mathrm{PhD}$ thesis]. Mosonmagyaróvár: ATE Mezőgazdasági Kar; 1985.

20. Györffy L. A nagy ínség 1863-ban. Túrkeve: Daróczy Antal Könyvnyomdája; 1931. (A Túrkevei Hírlap melléklete).

21. Molnár V. A. Növényi ínségeledelek. Természet Világa. 2010;141(11):514-516.

22. Kardos L. Az Örség népi táplálkozása: tanulmányok az Örség monográfiájához. Budapest: Államtudományi Intézet Táj- és Népkutató Osztálya; 1943.

23. Bödei J. Adatok Zalabaksa gyüjtögető gazdálkodásához. Néprajzi Értesítő. 1943;2:69-96.

24. Takáts G. Somogyi pásztorvilág. Kaposvár: Somogy Megyei Múzeumok Igazgatósága; 1986.

25. Füvesy A. Gyüjtögetés és erdőélés Mozsgó környékén. Tiscium. 1997;10:207-217.

26. Müller G. Gyüjtögető életmód szőkédi hagyományai. Budapest: Baranya Megyei Tanács V. B. Művelődésügyi Osztálya; 1973. (Baranyai Művelődés; vol 3).

27. Nagy R. Adatok a Baranyamegyei nagyváty növényekkel kapcsolatos szokásaihoz és néphagyományaihoz. Magyar Népnyelv. 1942;4:268-308.

28. Szigeti A. Népi konyha. Dunántúli tájak ételei. Ételkészítés alapanyagai tájanként. Budapest: Planétás Kiadó; 1999.

29. Táplálkozás adattári adatok Zengővárkony, Bonyhád, Szigetvár. Inv. No.: $7-75 ; 613-82 ; 255-80$. Database of the Ethnography Department of the Janus Pannonius Museum, Pécs.

30. Pethő M. Győr-Moson-Sopron megye XX. századi népi táplálkozásának rövid jellemzése. Arrabona. 36(1-2):137-150.

31. Dantér I. Zsákmányoló gazdálkodás Farkasdon és Negyeden. In: Dantér I, editor. Hagyományos gazdálkodás a Kisalföld északi részén: Farkasd, Negyed. Galánta: Galántai Honismereti Múzeum; 2005. p. 51-73.

32. Zentai J. Egy letűnt életforma képviselője az Ormánságban. Janus Pannonius Múzeum Évkönyve. 1965;11:181-203.

33. Kiss Z. G. Zsákmányoló foglalkozások: Halászat gyüjtögetés. In: Kiss Z. G, editor. A régi Vajszló: 1244-1849. Pécs: Vajszló Nagyközség Önkormányzata; 1994. p. 174-179.

34. Andrásfalvy B. A Duna mente népének ártéri gazdálkodása : ártéri gazdálkodás Tolna és Baranya megyében az ármentesítési munkák befejezése elott. Budapest: Ekvilibrium; 2007.

35. Lábadi K. Kopács, a víz melletti falu. Budapest: Horvátországi Magyarok Demokratikus Közössége; 1994.

36. Vajkai A. A gyüjtögető gazdálkodás Cserszegtomajon. Néprajzi Értesítő. 1941;23:231-258.

37. Hegyi I. Gyüjtögető gazdálkodás az északkeleti Bakonyban. Ethnographia. 1970;2-4:442-452.

38. Fehér J. Adatok Bernecebaráti gyűjtögető és zsákmányoló gazdálkodásához. Néprajzi Értesítő. 1957;2(3-4):267-292.

39. Gunda B. A gyüjtögető gazdálkodás emlékei egy gerecsehegységi tót faluban. Ethnographia. 1938;49(1-2):213-214.

40. Barsi E. Sály : egy bükkalji falu a hagyományos gazdálkodás idején. Miskolc: Herman Ottó Múzeum; 1987. (Néprajzi Kiadványa; vol 17).

41. Kóczián G. Etnobotanikai vizsgálatok Répáshután. Répáshuta, egy szlovák falu a Bükkben. Miskolc: Herman Ottó Múzeum; 1984. (Néprajzi
Kiadványa; vol 8).

42. Ujváry Z. A vadontermő növények szerepe a táplálkozásban az abaújzempléni hegyvidéken. Néprajzi Értesítő. 1957;39:231-243.

43. C. Schwalm E. Gyüjtögetés, mint nyersanyagkiegészítő. In: Bakó F, editor. Palócok III. Eger: Heves Megyei Múzeumok Igazgatósága; 1989. p. 417-423.

44. Paládi-Kovács A. A Barkóság és népe. Miskolc: Herman Ottó Múzeum; 2006.

45. Pálosné Nagy R. Az erdő hatása a gyöngyössólymosi emberek életére. Mátrai tanulmányok. 2000;

46. Sinkó R. Adatok Füzesgyarmat népi táplálkozáshoz. Békés Megyei Múzeumok Közleményei. 1996;16:267-288.

47. Petercsák T. Hegyköz. Miskolc: Herman Ottó Múzeum; 1978. (Borsodi Kismonográfiák; vol 6).

48. Márkus M. Gyüjtögetés a Csermosnya völgy. Néprajzi Értesítő. 1941;33(2):173-177.

49. Újváry Z. Vadon termő növények a táplálkozásban. In: Újváry Z, editor. Népi táplálkozás három Gömöri völgyben. Debrecen: Kossuth Lajos Tudományegyetem Néprajzi Tanszéke; 1991. p. 33-60. (Gömör néprajza; vol 29).

50. Zsupos Z. Dél-Gömör gyüjtögető gazdálkodása. Debrecen: Kossuth Lajos Tudományegyetem Néprajzi Tanszék; 1987. (Gömör Néprajza; vol 10).

51. Bodnár M. Adatok Tornaújfalu népi táplálkozásához. Miskolc: Herman Ottó Múzeum; 1988. (Gömör néprajza; vol 25-26).

52. Bődi E. A vadon flórája a gömöri magyarok táplálkozási kultúrájában. Tiscium. 1999;11:73-82.

53. Gunda B. Egy kárpátaljai magyar falu ethnobotanikája. Nyíregyházi Jósa András Múzeum Évkönyve. 1980;

54. Takács P, Udvardi I. Adalékok a Bereg, Ugocsa és Ung vármegyék lakóinak 18. századi erdőélési szokásaihoz. Hermann Ottó Múzeum Évkönyve. 1996;33-34:213-247.

55. Hanusz I. Füben, fában. Budapest: Atheneum Kiadó; 1905.

56. Kiss L. A nyírvíz. A Földgömb. 1930;1:1-6.

57. Fazekas M. Karcag népi táplálkozása. Debrecen: KLTE; 1994. (Studia Folkloristica et Ethnographica).

58. Ecsedi I. A debreceni és tiszántúli magyar ember táplálkozása. Debrecen: A Debreceni Déri Múzeum Évkönyve; 1934.

59. Bencsik J. A gyüjtögető gazdálkodás emlékei a Tisza mentén. A Hajdúsági Múzeum évkönyve. 1973;1:111-126.

60. T. Bereczky I. Gyüjtögető élelemszerzés. In: Barna G, editor. Csépa. Tanulmányok egy alföldi palóc kirajzás népéletéből. Eger-Szolnok: Damjanich János Múzeum; 1982.

61. T. Bereczky I. Népi táplálkozás Szolnok megyében. Kossuth Lajos Tudományegyetem Néprajzi Tanszékenek; 1986. (Studia Folkloristica et Ethnographica; vol 19).

62. Varga G. A népi táplálkozás Hajdú-Bihar megyében a XX. század elso felében. Debrecen: Hajdú-Bihar Megyei Múzeumi Igazgatóság; 1993. (A Hajdú-Bihar Megyei Múzeumok Közleményei; vol 52).

63. Palatinus A. "Kész az étel, gyé kend má önni”. Hagyományos táplálkozás a muzslyai házban. Népi táplálkozás a XX. században. Létünk. 2002;32(3-4):126-136.

64. Györffy I. Viricselés a Székelyföldön. Ethnographia. 1937;48(2):205-220.

65. Gunda B. A gyüjtögető életmód emlékei a Gyalui havasokban. Budapest: Tankönyvkiadó; 1960. (Muveltség és hagyomány).

66. Gunda B. Tejoltó növények a Kárpátokban. Ethnographia. 1967;78:161-175.

67. Gub J. Adatok a Nagy-Homoród és a Nagy-Küküllő közötti terület népi növényismeretéhez. Néprajzi Látóhatár. 1993;1-2:95-110.

68. Székely F. Népi gyüjtögetés Vadasdon. Kriza János Néprajzi Társaság Évkönyve. 1998;6:281-285.

69. Tarisznyás M. A gyüjtögető gazdálkodás hagyományai Gyergyóban. In: Népismereti dolgozatok. Bucharest: Kriterion; 1978. p. 25-33.

70. Rab J. Népi növényismeret a Gyergyói-medencében. Csíkszereda: PallasAkadémia Kiadó; 2001. 
71. Vita Z. Tápláléknövények gyüjtése Nagyenyed környékén. In: Népismereti dolgozatok. Bucharest: Kriterion; 1994. p. 44-47.

72. Kruzslits I. Az erdő adta javak a kalotaszegi Kiskapus gyüjtögető gazdálkodásában. Tisicum. 2007;16:115-119.

73. Mihalescu L, Vosgan Z, Rosca OM, Danci O. The ornamental, aromatic and edible vegetal species from Lapus region (Transylvania). In: 19th EVS Workshop. Flora, vegetation, environment and landuse at large scale Congress. 29 April-2 May, 2010, Univeristy of Pecs, Hungary. Pécs: Univeristy of Pécs; 2010. p. 66.

74. Papp N. Etnobotanikai adatok Erdélyből 2007-2012 között. Úz-völgye (Csinód Egerszék), Gyimes, Erdővidék (Nagybacon, Kisbacon, Uzonka) Homoródkarácsonyfalva, Lövéte [Manuscript]. 2012.

75. Babai D. Vadon élő tápláléknövények Gyimesben - Etnobotanikai gyüjtések [Manuscript]. Budapest: MTA Research Centre for the $\mathrm{Hu}-$ manities; 2012.

76. F. Várózi Z. A bukovinai székelyek táplálkozása 1958-1961 közötti gyüjtés alapján. [Manuscript]. Inv. No.: I./ 736-80. Database of the Ethnography Department of the Janus Pannonius Museum, Pécs. 1961.

77. Grynaeus T, Szabó LG. A bukovinai hadikfalvi székelyek növényei. Kriza János Néprajzi Társaság Évkönyve. 2002;10:153-246.

78. Molnár Z, editor. 34 magyar botanikus gyerekkori vadnövény csemegéi [Manuscript]. Vácrátót: Centre for Ecology Hungarian Academy of Sciences; 2012.

79. Łuczaj ŁJ. Plant identification credibility in ethnobotany: a closer look at Polish ethnographic studies. J Ethnobiol Ethnomed. 2010;6(1):36. http:// dx.doi.org/10.1186/1746-4269-6-36

80. Łuczaj $Ł$. Changes in the utilization of wild green vegetables in Poland since the 19th century: a comparison of four ethnobotanical surveys. J Ethnopharmacol. 2010;128(2):395-404. http://dx.doi.org/10.1016/j. jep.2010.01.038

81. Łuczaj ŁJ, Kujawska M. Botanists and their childhood memories: an underutilized expert source in ethnobotanical research. Bot J Linn Soc. 2012;168(3):334-343. http://dx.doi.org/10.1111/j.1095-8339.2011.01205.x

82. Łuczaj Ł. Ethnobotanical review of wild edible plants of Slovakia. Acta Soc Bot Pol. 2012;81(4):245-255. http://dx.doi.org/10.5586/asbp.2012.030

83. Kalle R, Sõukand R. Historical-ethnobotanical review of wild edible plants of Estonia, 1770s-1960s. Acta Soc Bot Pol. 2012;81(4):271-281. http:// dx.doi.org/10.5586/asbp.2012.033

84. Łuczaj ŁJ, Svanberg I, Köhler P. Marsh woundwort, Stachys palustris L. (Lamiaceae): an overlooked food plant. Genet Resour Crop Evol. 2011;58(5):783-793. http://dx.doi.org/10.1007/s10722-011-9710-9

85. Czúcz B, Gathman JP, McPherson GR. The impending peak and decline of petroleum production: an underestimated challenge for conservation of ecological integrity. Conserv Biol. 2010;24(4):948-956. http://dx.doi. org/10.1111/j.1523-1739.2010.01503.x

86. Zilahy Á. Valódi magyar szakácskönyv. Budapest: A Magyar nők Lapjának Kiadóhivatala; 1892.

87. Szabó György bükki füvesember weboldala [Internet]. 2012 [cited 2012 Aug 1]; Available from: http://www.gyorgytea.hu 